\title{
Generalized Lamé functions. II. Hyperbolic and trigonometric specializations
}

\author{
S. N. M. Ruijsenaars \\ Centre for Mathematics and Computer Science, P.O. Box 94079, \\ 1090 GB Amsterdam, The Netherlands
}

(Received 24 April 1998; accepted for publication 17 September 1998)

\begin{abstract}
In Part I [J. Math. Phys. 40, 1595 (1999)] we studied eigenfunctions of the quantum dynamics that defines the two-particle relativistic Calogero-Moser system with elliptic interaction. In the present paper we consider the same system with hyperbolic and trigonometric interactions. In these special regimes the eigenfunctions are shown to admit an elementary representation that is far more explicit than the "zero representation" of Part I. In particular, the new representation can be exploited to prove that the hyperbolic eigenfunctions can be chosen to be symmetric under interchanging position and momentum variables (self-duality). In the trigonometric case duality properties are derived, too, and several orthogonality and completeness results are obtained. (C) 1999 American Institute of Physics.

[S0022-2488(99)02502-5]
\end{abstract}

\section{INTRODUCTION}

In the preceding paper ${ }^{1}$ (henceforth denoted by $\mathrm{I}$ ) we obtained and studied joint eigenfunctions of two commuting analytic difference operators (A $\Delta \mathrm{OS}) A_{+}$and $A_{-}$[given by Eq. (1.15) of I or I(1.15)]. The coefficients of these $\mathrm{A} \Delta \mathrm{O}$ s are, in essence, elliptic. More precisely, both $\mathrm{A} \Delta \mathrm{O}$ $A_{\delta}$ have meromorphic coefficients with real period $\pi / r, r>0$, and imaginary quasi-period $i a_{\delta}$, $a_{\delta}>0, \delta=+,-$. In the present paper we study hyperbolic and trigonometric specializations of the operators and functions introduced in I, referring the reader to the Introduction of I for a description of the context from which the pertinent operators arise, their connection to the Lamé operator, and literature dealing with the subject area involved.

On the one hand, the results obtained in this paper illuminate the elliptic regime, inasmuch as various questions left open in I can be answered for the hyperbolic and trigonometric regimes. On the other hand, the special cases are of independent interest, and have some remarkable features no longer present at the elliptic level. We study the hyperbolic case in Secs. II and III, the trigonometric one in Sec. IV. Though we begin each section by indicating how the zero representation of the eigenfunctions obtained in I can be adapted, we need not and will not use these results. Indeed, we reobtain the zero representation from a second one that is quite elementary and explicit. More generally, this paper is largely independent of I, especially as concerns the hyperbolic case.

We proceed by sketching our hyperbolic results, turning to trigonometric results towards the end of this Introduction. For $r=0$ the commuting $A \Delta O$ s $\mathrm{I}(1.15)$ reduce to

$$
A_{\delta}(b)=\frac{s_{\delta}(x-i b)}{s_{\delta}(x)} T_{i a_{-\delta}}+(i \rightarrow-i), \quad \delta=+,-,
$$

where

$$
\left(T_{\alpha} f\right)(x)=f(x-\alpha), \quad \alpha \in \mathbb{C} .
$$

Here and below, we use the notation

$$
s_{\delta}(x)=\sinh \left(\pi x / a_{\delta}\right), \quad c_{\delta}(x)=\cosh \left(\pi x / a_{\delta}\right), \quad e_{\delta}(x)=\exp \left(\pi x / a_{\delta}\right), \quad \delta=+,-
$$


[We should point out that our hyperbolic $s_{\delta}$-function differs from the hyperbolic specialization of our elliptic $s_{\delta}$-function by a factor $a_{\delta} / \pi$; cf. $\mathrm{I}(1.11)$ and $\mathrm{I}(1.8)$. Though this may give rise to confusion, we are opting for this abuse of notation in order to minimize clutter from constants.]

Unless explicitly mentioned otherwise, we choose the parameters occurring here in the hyperbolic domain

$$
\mathcal{H} \equiv\left\{\left(a_{+}, a_{-}, b\right) \mid a_{+}, a_{-}>0, b \in \mathbb{R}\right\}
$$

This ensures that the Hamiltonians

$$
H_{\delta}(b) \equiv\left(\frac{s_{\delta}(x-i b)}{s_{\delta}(x)}\right)^{1 / 2} T_{i a_{-\delta}}\left(\frac{s_{\delta}(x+i b)}{s_{\delta}(x)}\right)^{1 / 2}+(i \rightarrow-i), \quad \delta=+,-
$$

are formally self-adjoint. The latter are related to the $\mathrm{A} \Delta \mathrm{Os} A_{\delta}$ by the similarity

$$
H_{\delta}=w(x)^{1 / 2} A_{\delta} w(x)^{-1 / 2} \text {. }
$$

Here, $w\left(a_{+}, a_{-}, b ; x\right)$ is the hyperbolic weight function studied in Ref. 2; cf. also I(1.16), I(1.17).

Save for the functional-analytic results in Sec. IV of I, it is straightforward to specialize the results in $I$ to the hyperbolic regime. As a matter of fact, considerable simplification occurs at several places, in particular, in Appendix B of $\mathrm{I}$, where uniqueness of joint eigenfunctions is studied.

The latter uniqueness results are the only ones needed, however. Indeed, we start from a representation of the joint eigenfunctions that looks quite different from the zero representation obtained in I. This new representation holds true for the dense subset of $\mathcal{H}$ (1.4) given by

$$
\mathcal{D}_{\text {hyp }} \equiv\left\{\left(a_{+}, a_{-}, b\right) \mid a_{+}, a_{-}>0, b=k a_{+}+l a_{-}, k, l \in \mathbb{Z}\right\} .
$$

Thus no $(k, l)$-dependent restriction on $\left(a_{+}, a_{-}\right)$occurs, by contrast to the dense subset $\mathcal{D} \subset \mathcal{D}_{\text {hyp }}$ that arises upon specializing the zero representation in $\mathrm{I}$.

More is true: We could even allow $a_{+}$and $a_{-}$to be arbitrary numbers in $\mathrm{C}^{*}$. Similarly, $x$ and the spectral variable $p$ may be chosen complex. Indeed, for a fixed $b$ of the form $k a_{+}+l a_{-}$, $k, l \in \mathbb{Z}$, we obtain functions $M_{k, l}\left(a_{+}, a_{-} ; \pm x, p\right)$ that are one-valued analytic functions in all of their four arguments, and that satisfy the joint eigenfunction equations

$$
A_{\delta} M=2 c_{\delta}(p) M, \quad \delta=+,-.
$$

The variable $p$ is related to the variable $y$ used in I via

$$
p=a_{+} a_{-} y / \pi
$$

This rescaling ensures that the eigenfunctions are symmetric under interchanging $x$ and $p$ (selfduality). To be sure, this property is by no means evident from the explicit formulas-it is the quantum translation of a classical self-duality property that is not manifest either, cf. Ref. 3. As it turns out, quantum self-duality boils down to some novel " $q$-identities" [viz., symmetry of the coefficients $c_{k l}^{(N)}(q)$ given by (2.2)-(2.5)].

To provide more perspective on the $b$-restriction in $\mathcal{D}_{\text {hyp }}(1.7)$, we would like to mention that the even linear combination,

$$
R_{k, l}\left(a_{+}, a_{-} ; x, p\right)=M_{k, l}\left(a_{+}, a_{-} ; x, p\right)+(x \rightarrow-x)
$$

admits an interpolation to all parameters in $\mathcal{H}$ (1.4). To be specific, there exists a joint $A_{\delta^{-}}$-eigenfunction $R\left(a_{+}, a_{-}, b ; x, p\right)$ that reduces to $R_{k, l}$ for $b=k a_{+}+l a_{-}$; it is meromorphic in $x$ and $p$ for fixed $\left(a_{+}, a_{-}, b\right) \in \mathcal{H}$ and real-analytic on $\mathcal{H}$ for fixed $x, p$ with $\operatorname{Re} x, \operatorname{Re} p \neq 0$. We already detailed this function in Subsection 6.3 of our lecture notes, Ref. 4 , and it will be further 
studied elsewhere. It is quite unclear whether the odd combination admits interpolation, too. (If so, it presumably has a quite different structure and weaker analyticity properties; cf. the pertinent discussion in I.)

Let us now describe the contents of Secs. II and III in some more detail. In Sec. II we study the special case where the coupling constant

$$
g \equiv b / a_{+}
$$

takes integer values. More precisely, we only study the choices

$$
b=(N+1) a_{+}, \quad N \in \mathbb{N} .
$$

For this special case the hyperbolic eigenfunctions and several salient features thereof were already presented in our survey, Ref. 5, but detailed proofs were not given there. In Sec. II we demonstrate various properties of an algebraic nature, but we relegate an account of orthogonality and completeness properties to another occasion.

Specifically, the joint $A_{\delta}$-eigenfunctions read

$$
M_{N+1,0}\left(a_{+}, a_{-} ; x, p\right) \equiv(-i)^{N+1}\left[P_{N}(x) P_{N}(p)\right]^{-1} K_{N}(x, p),
$$

with

$$
\begin{gathered}
P_{N}(x) \equiv \prod_{j=-N}^{N}\left[2 s_{-}\left(x+i j a_{+}\right)\right], \\
K_{N}(x, p) \equiv \exp \left(i \pi x p / a_{+} a_{-}\right) \sum_{k, l=0}^{N} c_{k l}^{(N)}(q) e_{-}((N-2 k) x+(N-2 l) p) .
\end{gathered}
$$

Here, the coefficients $c_{k l}$ depend only on $N$ and the phase factor

$$
q \equiv \exp \left(i \pi a_{+} / a_{-}\right)
$$

Explicitly, they are Laurent polynomials in $q$ with integer coefficients, given by (2.2)-(2.5).

Equivalently, the function $K_{N}(x, p)$ is a joint eigenfunction of the auxiliary $\mathrm{A} \Delta \mathrm{Os}$

$$
\begin{gathered}
B_{-}=\frac{s_{-}\left(x+i N a_{+}\right)}{s_{-}(x)} T_{i a_{+}}+(i \rightarrow-i), \\
B_{+}=(-)^{N} T_{i a_{-}}+(i \rightarrow-i),
\end{gathered}
$$

obtained by similarity transforming the $\mathrm{A} \Delta \mathrm{Os} A_{\delta}\left((N+1) a_{+}\right)$with $P_{N}(x)$. Observe that one of the two eigenvalue equations, viz.,

$$
B_{+} K_{N}(x, p)=2 c_{+}(p) K_{N}(x, p)
$$

is immediate from the structure (1.15) of $K_{N}$, independently of the choice of $c_{k l}$. With (2.2)-(2.5) in force, the second one (2.1) is proved in Theorem II.1, together with various other features of $K_{N}(x, p)$.

With these results at our disposal, we are in the position to make the connection to the seemingly different joint eigenfunctions arising upon hyperbolic specialization of Sec. II in I. Moreover, several uniqueness aspects can be clarified by adapting Theorem B.1 in I to the case at hand. Subsequently, we study the even combination $R_{N+1,0}\left(a_{+}, a_{-} ; x, p\right)(1.10)$ in Theorem II.2. [It is denoted $R_{N}(x, p)$ for brevity.] In particular, we show that this joint $A_{\delta}$-eigenfunction specializes to a polynomial in $c_{-}(x)$ for certain values of $p$. These results will be exploited for the trigonometric regime (Sec. IV). 
The third and last theorem of Sec. II concerns the case of a rational quotient $a_{+} / a_{-}$. It throws new light on the zero representation, and is also a crucial input for Sec. III.

In the latter section we obtain joint $A_{\delta^{-}}$and $H_{\delta^{-}}$eigenfunctions for arbitrary parameters in $\mathcal{D}_{\text {hyp }}$ (1.7), but just as in Sec. II it is convenient to use an auxiliary pair of $\mathrm{A} \Delta \mathrm{Os} B_{+}, B_{-}$as a starting point. These $A \Delta \mathrm{Os}$ are defined for $b$ of the form

$$
b_{+-} \equiv\left(N_{+}+1\right) a_{+}-N_{-} a_{-}, \quad N_{+}, N_{-} \in \mathbb{N},
$$

by similarity transforming $A_{+}, A_{-}(1.1)$ with $P_{N_{+}}(x)$ (1.14). Explicitly, this yields

$$
B_{\delta}=(-)^{N_{\delta}} \frac{s_{\delta}\left(x+i N_{-\delta} a_{-\delta}\right)}{s_{\delta}(x)} T_{i a_{-\delta}}+(i \rightarrow-i), \quad \delta=+,-
$$

[Note this reduces to $(1.17),(1.18)$ for $N_{+}=N, N_{-}=0$, and $\delta=-,+$, as should be the case, of course.]

Using the functions $K_{N}(x, p)$ from Sec. II as building blocks, joint $B$-eigenfunctions are readily constructed. By virtue of (the hyperbolic specialization of) Theorem B.1 in I, the joint $B_{\delta}$-eigenspace associated with eigenvalues $2 c_{\delta}(p)$ is two-dimensional for $a_{+} / a_{-}$irrational. Now it is clear that the $b$-values (1.20) with $a_{+} / a_{-}$irrational already give rise to a dense subset of the hyperbolic parameter domain $\mathcal{H}$ (1.4). Moreover, the $\mathrm{A} \Delta \mathrm{Os} B_{\delta}(1.21)$ may be reinterpreted as specializations of the $\mathrm{A} \Delta \mathrm{OS}$

$$
B_{\delta}(b)=\frac{s_{\delta}\left(x-i \delta\left(b-a_{+}\right)\right)}{s_{\delta}(x)} T_{i a_{-\delta}}+(i \rightarrow-i), \quad \delta=+,-,
$$

which are defined for all of $\mathcal{H}$. [By contrast, the elliptic generalizations $\mathrm{I}(4.7)$ do not admit a continuous interpolation to the whole elliptic parameter domain.]

On the other hand, the joint $B_{\delta}$-eigenfunctions exhibit an infinite-dimensional ambiguity already for the $b$-values $b_{+-}(1.20)$ and rational $a_{+} / a_{-}$. This provides an example demonstrating that the absence of interpolation ambiguities cannot follow from general arguments (as one might believe). But the ambiguity exhibited by the joint $B_{\delta}$-eigenfunctions does not occur for the joint $H_{\delta^{-}}$and $A_{\delta^{-}}$eigenfunctions. Indeed, we show that for rational $a_{+} / a_{-}$the infinity of distinct $(k, l) \in \mathbb{Z}^{2}$ yielding the same $b=k a_{+}+l a_{\text {- }}$ gives rise to an infinity of distinct representations for the same function.

In order to arrive at the latter conclusions, we need as technical input Theorem III.1, which deals with the case of rational $a_{+} / a_{-}$. The joint $H_{\delta^{-}}$and $A_{\delta}$-eigenfunctions $F(\Xi ; x, p)$ and $M(\Xi ; x, p)$ for arbitrary $\Xi \in \mathcal{D}_{\text {hyp }}$ (1.7) are further studied in Theorems III.2 and III.3, respectively; the meromorphic functions $M_{k, l}\left(a_{+}, a_{-} ; \pm x, p\right)$ mentioned above are equal to $M\left(a_{+}, a_{-}, b ; \pm x, p\right)$ for $b=k a_{+}+l a_{-}$.

Let us now turn to the trigonometric regime, studied in Sec. IV. This arises from the elliptic regime by sending one of the two imaginary periods $i a_{+}, i a_{-}[\mathrm{cf} . \mathrm{I}(1.11)]$ to $i \infty$. We will take $a_{-}$ to $\infty$ and trade $a_{+}$for a new parameter $\beta$. Of course, the real period $\pi / r$ is kept fixed. Thus, we arrive at the trigonometric parameter domain

$$
\mathcal{T} \equiv\{(r, \beta, b) \mid r, \beta>0, b \in \mathbb{R}\} .
$$

Obviously, the elliptic A $\Delta \mathrm{Os} H_{+}$and $A_{+}$have no limits for $a_{-} \rightarrow \infty$. Therefore we are left with

$$
A(b)=\frac{\sin r(x-i b)}{\sin r x} T_{i \beta}+(i \rightarrow-i)
$$




$$
H(b)=\left(\frac{\sin r(x-i b)}{\sin r x}\right)^{1 / 2} T_{i \beta}\left(\frac{\sin r(x+i b)}{\sin r x}\right)^{1 / 2}+(i \rightarrow-i) .
$$

These $\mathrm{A} \Delta \mathrm{O}$ s are related by the similarity

$$
A=w(x)^{-1 / 2} H w(x)^{1 / 2},
$$

with $w(r, \beta, b ; x)$ the trigonometric weight function from Ref. 2. Now the parameter $b$ is of the form $k a_{+}+l a_{-}, k, l \in \mathbb{Z}$, for all of the eigenfunctions in I. Thus, we need to choose $l=0$ for $b$ to remain finite as $a_{-} \rightarrow \infty$. Accordingly, we only obtain eigenfunctions for the $\mathcal{T}$-subset

$$
\mathcal{D}_{\text {trig }} \equiv\{(r, \beta, b) \mid r, \beta>0, b=k \beta, k \in \mathbb{Z}\}
$$

which is no longer dense. Just as in I, all of the pertinent functions are also eigenfunctions of the quasi-periodicity $\mathrm{A} \Delta \mathrm{O}$

$$
Q \equiv T_{\pi / r}+T_{-\pi / r}
$$

Our trigonometric joint $(A, Q)$-eigenfunctions are obtained via analytic continuation of their hyperbolic counterparts from Sec. II. Besides the zero representation obtained by specializing Sec. II in paper I to the trigonometric regime, we therefore get a second, far more accessible, representation.

We begin Sec. IV by detailing the latter, and then clarify its relation to the zero representation. In the remainder of Sec. IV we deal with various functional-analytic aspects. Correspondingly, the spectral variable is discretized, and we wind up with Hilbert space eigenfunctions that are essentially $q$-Gegenbauer polynomials, with $q=\exp (-2 \beta r)$. To our knowledge, our two representations are new even in this well-studied case.

By contrast to Secs. II, III, and the first part of Sec. IV, which are largely self-contained, the remainder of Sec. IV involves various features and issues already encountered in Sec. IV of I. In particular, the drastic simplification arising in the trigonometric case allows us to answer some questions that we left open in the elliptic setting. These questions can be studied by choosing $k$ negative in (1.27).

\section{THE HYPERBOLIC INTEGER- $g$ CASE}

The results of this section have already been summarized in some detail in the Introduction, and we will freely use the notation and operators introduced there.

We begin by recalling that in Sec. II of I we also restricted attention to the integer $g$ case (1.12). Now when one replaces the function $s(r, a ; x)$ from I by its hyperbolic counterpart $(a / \pi) \sinh (\pi x / a)$, then it is straightforward to adapt the arguments and results that can be found in Sec. II of I. There is only one minor snag in the reasoning below I(2.13): A nonconstant hyperbolic function may have one or no pole in a period strip; cf. the functions $\operatorname{cotanh}(x)$ and $\cosh (x)$. The pertinent hyperbolic function $E(x) \mathrm{I}(2.8)$, however, has finite and equal limits for $\operatorname{Re} x$ $\rightarrow \pm \infty$. Therefore, the usual residue argument for elliptic functions can be easily adapted to exclude the presence of only one pole in the period strip.

The results of this section go far beyond those of Sec. II in I, however. The crux is that the eigenfunctions and eigenvalues admit a simpler and much more explicit form in the hyperbolic setting, without restrictions on the spectral variable $y$ and the pertinent parameters. In particular, this enables us to shed more light on the "zero representation" I(2.34) of the eigenfunctions. As will be shown, the latter structure of the eigenfunctions is a consequence of the eigenfunction representation employed in this section, but various features obtained below are invisible from $I(2.34)$. For example, the spectral variable $y$ appears to be on a very different footing from the variable $x$, whereas it will turn out that $x$ and the rescaled spectral variable $p$ (1.9) play symmetric roles. 
We proceed by detailing the joint eigenfunctions $K_{N}(x, p)(1.15)$ of the $\mathrm{A} \Delta \mathrm{Os} B_{-}(1.17)$ and $B_{+}(1.18)$ with eigenvalues $2 c_{\delta}(p), \delta=+,-$. As already pointed out, the eigenvalue equation (1.19) is satisfied irrespective of the choice of $c_{k l}$. It will be shown later on, however, that the coefficients are uniquely determined up to an overall $q$-dependent scale factor by requiring

$$
B_{-} K_{N}(x, p)=2 c_{-}(p) K_{N}(x, p),
$$

and continuity in $q$.

In order to specify $c_{k l}$, we introduce $N$-element subsets $I_{k}^{(N)}$ of the $2 N$-element set $\{-N, \ldots,-1,1, \ldots, N\}$, as follows:

$$
I_{k}^{(N)} \equiv\{-N, \ldots,-N+k-1, \ldots, k+1, \ldots, N\}, \quad k=0, \ldots, N .
$$

Now we put

$$
\begin{gathered}
s_{k l}^{(N)}(w) \equiv \sum_{\substack{i_{1}<\ldots<i_{k} \\
i_{m} \in I_{l}^{(N)}}} w^{i_{1}+\cdots+i_{k}}, \\
c_{l}^{(N)}(w) \equiv \sum_{\substack{i_{1}<\ldots<i_{l} \\
i_{m} \in I_{0}^{(N)}}} w^{i_{1}+\cdots+i_{l}}, \\
c_{k l}^{(N)}(q) \equiv(-)^{k+l} q^{N(N+1) / 2} s_{k l}^{(N)}\left(q^{-2}\right) c_{l}^{(N)}\left(q^{-2}\right) .
\end{gathered}
$$

(Here, empty sums equal 1 by definition.) For later use we also introduce polynomials

$$
Q_{l}^{(N)}(u) \equiv \sum_{k=0}^{N}(-)^{k} s_{k l}^{(N)}(w) u^{k}=\prod_{i \in I_{l}^{(N)}}\left(1-w^{i} u\right)
$$

With these definitions in place, we are going to prove that (2.1) holds true. Before doing so, however, we specify the cases $N=0, \ldots, 3$, exemplifying the above notation:

$$
\begin{gathered}
(N=0) \quad c_{00}=1, \\
(N=1) \quad c_{00}=c_{11}=q, \quad c_{01}=c_{10}=-q^{-1}, \\
(N=2) \quad c_{00}=c_{22}=q^{3}, \quad c_{02}=c_{20}=q^{-3}, \\
c_{01}=c_{10}=c_{12}=c_{21}=-q-q^{-1}, \quad c_{11}=q^{5}+q^{3}+q^{-3}+q^{-5}, \\
(N=3) \quad c_{00}=c_{33}=q^{6}, \quad c_{03}=c_{30}=-q^{-6}, \\
c_{02}=c_{20}=c_{13}=c_{31}=-\bar{c}_{32}=-\bar{c}_{23}=-\bar{c}_{01}=-\bar{c}_{10}=1+q^{-2}+q^{-4}, \\
c_{11}=c_{22}=-\bar{c}_{12}=-\bar{c}_{21}=q^{10}+q^{8}+q^{6}+1+2 q^{-2}+2 q^{-4}+q^{-6} .
\end{gathered}
$$

Note that, more generally, the coefficients are Laurent polynomials in $q$ with integer coefficients for arbitrary $N \in \mathbb{N}$. The symmetry properties

$$
\dot{c}_{k l}=c_{l k}=c_{N-k, N-l}=(-)^{N} \bar{c}_{N-k, l}, \quad k, l=0, \ldots, N,
$$

exhibited by these special cases are, in fact, valid for arbitrary $N$; they are equivalent to the symmetry properties (2.13)-(2.15) in the following theorem. 
Theorem 1I.1: With $c_{k l}$ defined by (2.2)-(2.5), the function $K_{N}(x, p)(1.15)$ satisfies the $A \Delta E$

$$
s_{-}\left(x+i N a_{+}\right) F\left(x-i a_{+}\right)+s_{-}\left(x-i N a_{+}\right) F\left(x+i a_{+}\right)=2 s_{-}(x) c_{-}(p) F(x) .
$$

It has the symmetry properties

$$
\begin{gathered}
K_{N}(x, p)=K_{N}(p, x), \\
K_{N}(x, p)=K_{N}(-x,-p), \\
K_{N}(x, p)=(-)^{N} \bar{K}_{N}(-x, p), \quad x, p \in \mathbb{R},
\end{gathered}
$$

and satisfies

$$
K_{N}\left(x, i \delta N a_{+}\right)=(2 i)^{N} \prod_{k=N+1}^{2 N} \sin \left(\pi k a_{+} / a_{-}\right), \quad \delta=+,-
$$

Now assume

$$
k a_{+} \notin \mathbb{N} a_{-}, \quad k=1, \ldots, 2 N
$$

Then one has

$$
K_{N}\left(x, i \delta(N-l) a_{+}\right)=i^{N} B_{l}^{(N)}\left(c_{-}(x)\right), \quad l=0, \ldots, N, \quad \delta=+,-,
$$

where $B_{l}^{(N)}(u)$ is a polynomial of degree $l$ and parity $(-)^{l}$ with real coefficients.

Proof: For $N=0$ we have

$$
K_{0}(x, p)=\exp \left(i \pi x p / a_{+} a_{-}\right)
$$

and all assertions are immediate. Assuming $N \in \mathbb{N}^{*}$ from now on, we find it convenient to rewrite $K_{N}(x, p)$ as

$$
K_{N}(x, p)=K_{0}(x, p) e_{-}(N x+N p) S_{N}\left(q ; e_{-}(-2 x), e_{-}(-2 p)\right),
$$

with

$$
S_{N}(q ; r, t)=\sum_{k, l=0}^{N} c_{k l}^{(N)}(q) r^{k} t^{l}
$$

Now we fix $N \in \mathbb{N}^{*}$ and suppress the dependence on $N$ wherever this does not give rise to confusion.

We first view the general form (2.20)-(2.21) of $K(x, p)$ as an Ansatz for solving the $\mathrm{A} \Delta \mathrm{E}$ (2.12), so as to arrive at a system of equations for the coefficients $c_{k l}$. We then study this system in its own right before proving that it is satisfied by the above coefficients (2.5). The general insights thus obtained will be crucial for later purposes.

Accordingly, we plug (2.20) into (2.12), and cancel factors to obtain

$$
\begin{aligned}
& {\left[q^{N} e_{-}(x)-q^{-N} e_{-}(-x)\right] e_{-}(p) q^{-N} S\left(q^{2} e_{-}(-2 x), e_{-}(-2 p)\right)} \\
& \quad+\left[q^{-N} e_{-}(x)-q^{N} e_{-}(-x)\right] e_{-}(-p) q^{N} S\left(q^{-2} e_{-}(-2 x), e_{-}(-2 p)\right) \\
& \quad=\left[e_{-}(x)-e_{-}(-x)\right]\left[e_{-}(p)+e_{-}(-p)\right] S\left(e_{-}(-2 x), e_{-}(-2 p)\right) .
\end{aligned}
$$

Multiplying by $e_{-}(-x-p)$ and using $(2.21)$, this can be rewritten as 


$$
\begin{gathered}
\left(1-w^{N} r\right) \sum_{k, l=0}^{N} c_{k l} w^{-k} r^{k} t^{l}+\left(1-w^{-N} r\right) t \sum_{k, l=0}^{N} c_{k l} w^{k} r^{k} t^{l}-(1-r)(1+t) \sum_{k, l=0}^{N} c_{k l} r^{k} t^{l}=0 \\
w \equiv q^{-2}=\exp \left(-2 i \pi a_{+} / a_{-}\right) .
\end{gathered}
$$

Clearly, this is satisfied iff the coefficients $d_{m n}$ of the monomials $r^{m} t^{n}, m, n=0, \ldots, N+1$, vanish. The latter read

$$
d_{m n}=\left(1-w^{-N+m-1}\right) c_{m-1, n-1}+\left(1-w^{N-m+1}\right) c_{m-1, n}-\left(1-w^{m}\right) c_{m, n-1}-\left(1-w^{-m}\right) c_{m n} .
$$

We now study the system of equations $d_{m n}=0$ for the unknowns $c_{k l}$ under the side conditions

$$
c_{k l}=0, \quad k<0, \quad k>N, \quad l<0 .
$$

These conditions are obviously satisfied for the above coefficients, and they entail $d_{m n}=0$ for $m \leqslant 0, m \geqslant N+1$, and $n \leqslant-1$. (This is because the first two terms in brackets vanish for $m=N$ +1 and the last two for $m=0$.) Thus, we wind up with the system $d_{m n}=0$, where $m=1, \ldots, N$, $n \in \mathbb{N}$, for unknowns $c_{k l}$ in the vertical half-strip $k=0, \ldots, N, l \in \mathbb{N}$.

To avoid degeneracies, we now fix $a_{+}, a_{-} \in(0, \infty)$ such that $a_{+} l a_{-} \notin Q$. We claim that the solution to the system is then uniquely determined, provided we prescribe the numbers $c_{0 n}$ $\equiv b_{n}, n \in \mathbb{N}$, at the left boundary of the half-strip. To explain this, we observe that the system involves four lattice points on a plaquette. Thus, we can calculate successively $c_{m n}$ $=c_{10}, c_{20}, \ldots, c_{N 0}, c_{11}, \ldots c_{N 1}, c_{21}, \ldots$, etc. [Indeed, since $w^{m} \neq 1$ for $m \in \mathbb{Z}^{*}$, the term $\left(1-w^{-m}\right)$ in (2.24) is nonzero.] Hence our claim follows. In particular, there exists a uniquely determined solution to the system when we choose boundary coefficients

$$
b_{n} \equiv\left\{\begin{array}{l}
(-)^{n} w^{-N(N+1) / 4} \sum_{1 \leqslant i_{1}<\cdots<i_{n} \leqslant N} w^{i_{1}+\cdots+i_{n}}, \quad n=0, \ldots, N, \\
0, \quad n>N,
\end{array}\right.
$$

in accordance with (2.2)-(2.5).

The unicity of this solution will be crucial shortly, but we first prove that the unique solution is actually given by (2.5). Though this can be seen directly, it is somewhat simpler to recall that the solution property is equivalent to (2.23), and to observe that (2.23) holds iff the coefficients of the monomials $t^{n}, n=0, \ldots, N+1$, vanish. With $c_{k l}$ given by (2.5), the latter conditions can be written as

$$
\begin{array}{r}
\left(1-w^{N} r\right) b_{n} Q_{n}\left(w^{-1} r\right)+\left(1-w^{-N} r\right) b_{n-1} Q_{n-1}(w r)-(1-r)\left[b_{n} Q_{n}(r)+b_{n-1} Q_{n-1}(r)\right]=0, \\
n=0, \ldots, N+1,
\end{array}
$$

since we have

$$
b_{n} Q_{n}(r)=\sum_{m=0}^{N} c_{m n} r^{m}, \quad b_{n}=c_{0 n}
$$

cf. (2.6). The crux is that we may now cancel common factors in (2.27), which yields a recurrence relation for the boundary coefficients.

Specifically, taking first $n=0$ in (2.27) and noting

$$
Q_{0}(r)=\prod_{j=1}^{N}\left(1-w^{j} r\right)
$$


we deduce that (2.27) is satisfied. Similarly, (2.27) is satisfied for $n=N+1$. For $n \in\{1, \ldots, N\}$ we can cancel factors to obtain

$$
\begin{gathered}
b_{n}\left(1-w^{n} r\right)\left(1-w^{-N-1} r\right)+b_{n-1}\left(1-w^{N+1} r\right)\left(1-w^{-N+n-1} r\right) \\
-b_{n}(1-r)\left(1-w^{-N+n-1} r\right)-b_{n-1}(1-r)\left(1-w^{n} r\right)=0 .
\end{gathered}
$$

Simplifying this, we can divide by $w^{-N+n-1}-w^{n}$ to obtain

$$
\left(1-w^{-n}\right) b_{n}=\left(1-w^{N-n+1}\right) b_{n-1} .
$$

Thus, it remains to show that the boundary coefficients (2.26) satisfy this recurrence relation.

In order to prove this, we write the recurrence as

$$
\begin{array}{r}
\left(1-w^{-n}\right) \quad \sum_{1 \leqslant i_{1}<\cdots<i_{n} \leqslant N} w^{i_{1}+\cdots+i_{n}}+\left(1-w^{N-n+1}\right) \sum_{1 \leqslant i_{1}<\cdots<i_{n-1} \leqslant N} w^{i_{1}+\cdots+i_{n-1}}=0, \\
n=1, \ldots, N .
\end{array}
$$

Now we first handle the special case $n=N$. Then (2.32) reads

$$
\left(1-w^{-N}\right) w^{1+\cdots+N}+(1-w) w^{1+\cdots+N}\left(w^{-1}+w^{-2}+\cdots+w^{-N}\right)=0,
$$

which is clearly true. Next, we use induction on $N$. Thus we assume (2.32) holds when $N$ is replaced by $N-1$. Then we need only prove (2.32) for $n \in\{1, \ldots, N-1\}$. To this end we rewrite the first term on the lhs, using the induction hypothesis:

$$
\begin{aligned}
\left(1-w^{-n}\right)\left(\sum_{1 \leqslant i_{1}<\cdots<i_{n} \leqslant N-1} w^{i_{1}+\cdots+i_{n}}+w^{N} \sum_{1 \leqslant i_{1}<\cdots<i_{n-1} \leqslant N-1} w^{i_{1}+\cdots+i_{n-1}}\right) \\
=-\left(1-w^{N-n}\right) \sum_{1 \leqslant i_{1}<\cdots<i_{n-1} \leqslant N-1} w^{i_{1}+\cdots+i_{n-1}+\left(w^{N}-w^{N-n}\right)} \\
\quad \times \sum_{1 \leqslant i_{1}<\cdots<i_{n-1} \leqslant N-1} w^{i_{1}+\cdots+i_{n-1}} \\
=\left(w^{N}-1\right) \sum_{1 \leqslant i_{1}<\cdots<i_{n-1} \leqslant N-1} w^{i_{1}+\cdots+i_{n-1}} .
\end{aligned}
$$

Adding the second term, we obtain

$$
\begin{aligned}
& w^{N}\left(\sum_{1 \leqslant i_{1}<\cdots<i_{n-1} \leqslant N-1}-\sum_{0 \leqslant i_{1}<\cdots<i_{n-1} \leqslant N-1}\right) w^{i_{1}+\cdots+i_{n-1}} \\
& \quad+\left(\sum_{1 \leqslant i_{1}<\cdots<i_{n-1} \leqslant N}-\sum_{1 \leqslant i_{1}<\cdots<i_{n-1} \leqslant N-1}\right) w^{i_{1}+\cdots+i_{n-1}} \\
& \quad=-w^{N} \sum_{1 \leqslant i_{2}<\cdots<i_{n-1} \leqslant N-1} w^{i_{2}+\cdots+i_{n-1}+w^{N}} \sum_{1 \leqslant i_{1}<\cdots<i_{n-2} \leqslant N-1} w^{i_{1}+\cdots+i_{n-2}=0,}
\end{aligned}
$$

and so (2.32) follows.

The upshot is that $K(x, p)$ satisfies the $\mathrm{A} \Delta \mathrm{E}(2.12)$. To prove the symmetry properties (2.13)(2.15), we exploit the uniqueness of the solution to the system $d_{m n}=0$ with side conditions (2.25) and boundary condition (2.26). First, let us note that (2.13) is equivalent to symmetry of the 
coefficient matrix; cf. (1.15). Now it is clear from (2.2)-(2.5) that we have $c_{m 0}=c_{0 m}$ for $m$ $=0, \ldots, N$, so by uniqueness it suffices to show that the transposed matrix solves the system $d_{m n}$ $=0$, too.

In order to prove this, we use (2.26) to write the pertinent numbers $d_{m n}$ as

$$
\begin{aligned}
& b_{m-1}\left((-)^{n-1} s_{n-1, m-1}\left(1-w^{-N+m-1}\right)+(-)^{n} s_{n, m-1}\left(1-w^{N-m+1}\right)\right) \\
& -b_{m}\left((-)^{n-1} s_{n-1, m}\left(1-w^{m}\right)+(-)^{n} s_{n m}\left(1-w^{-m}\right)\right) .
\end{aligned}
$$

Now we deduce from the recurrence relation (2.31) that this expression vanishes iff

$$
w^{-N+m-1} s_{n-1, m-1}+s_{n, m-1}=s_{n m}+w^{m} s_{n-1, m} .
$$

From (2.3) we see that this amounts to

$$
\begin{gathered}
w^{-N+m-1} \sum_{\substack{i_{1}<\ldots<i_{n-1} \\
i_{l} \in I_{m-1}}} w^{i_{1}+\cdots+i_{n-1}}+\sum_{\substack{i_{1}<\ldots<i_{n} \\
i_{l} \in I_{m-1}}} w^{i_{1}+\cdots+i_{n}} \\
=\sum_{\substack{i_{1}<\ldots<i_{n} \\
i_{l} \in I_{m}}} w^{i_{1}+\cdots+i_{n}+w^{m}} \sum_{\substack{i_{1}<\ldots<i_{n-1} \\
i_{l} \in I_{m}}} w^{i_{1}+\cdots+i_{n-1}} .
\end{gathered}
$$

A moment's thought reveals that this is indeed true: both the lhs and rhs are equal to the sum

$$
\sum_{\substack{\left.i_{1}<\ldots<i_{n} \\ i_{1}, \ldots, N+m-1, m, \ldots, N\right\}}} w^{i_{1}+\cdots+i_{n}}
$$

Therefore, the self-duality relation $(2.13)$ is now proved.

Next, we demonstrate (2.14) and (2.15). Since (2.14) follows by combining (2.15) with the already proved symmetry property (2.13), it suffices to show (2.15). In view of (1.15) this amounts to $c_{k l}$ being equal to $(-)^{N} \bar{c}_{N-k, l}$, and since the coefficient matrix is symmetric we need only show

$$
c_{k l}=(-)^{N} \bar{c}_{k, N-l} \text {. }
$$

Now from (2.3) we deduce $\bar{s}_{k, N-l}=s_{k l}$, and from (2.4) we have

$$
\bar{c}_{N-l}=\sum_{1 \leqslant i_{1}<\cdots<i_{N-l} \leqslant N} w^{-\left(i_{1}+\cdots+i_{N-l}\right)}=w^{-(1+2+\cdots+N)} \sum_{1 \leqslant j_{1}<\cdots<j_{l} \leqslant N} w^{j_{1}+\cdots j_{l}}=q^{N(N+1)} c_{l} .
$$

Therefore, (2.40) is clear from (2.5).

In summary, we have now proved that $K(x, p)$ (1.15) satisfies (2.12)-(2.15), provided $a_{+} / a_{-} \notin \mathbb{Q}$. (Recall that the restriction was needed to ensure uniqueness of the solution to the coefficient system. To see why uniqueness breaks down otherwise, one need only inspect the special case $a_{+}=a_{-}$.) Since the coefficients $c_{k l}(q)$ are Laurent polynomials in $q$ $=\exp \left(i \pi a_{+} / a_{-}\right)$, the function $K(x, p)$ is well defined and continuous for all $a_{+}, a_{-} \in(0, \infty)$. Hence, it satisfies (2.12)-(2.15) for rational $a_{+} / a_{-}$, too.

We continue by proving (2.16). From (2.19)-(2.21) we have

$$
K\left(x,-i N a_{+}\right)=e_{-}(N x) e_{-}(N x) q^{-N^{2}} \sum_{k, l=0}^{N} c_{k l} e_{-}(-2 k x) q^{2 l N} .
$$

Using $c_{k l}=c_{l k}$ and recalling (2.2)-(2.6), this can be rewritten as 


$$
K\left(x,-i N a_{+}\right)=e_{-}(2 N x) q^{-N^{2}+N(N+1) / 2} \sum_{k=0}^{N}(-)^{k} c_{k} e_{-}(-2 k x) Q_{k}\left(q^{2 N}\right)
$$

The key point is now that $Q_{k}(u)$ vanishes for $u=q^{2 N}=w^{-N}$, unless $k=N$. [Indeed, $N$ belongs to $I_{k}^{(N)}$, save for $k=N$; cf. (2.2).] Hence we get

$$
K\left(x,-i N a_{+}\right)=(-)^{N} q^{-N^{2}-N(N+1) / 2} Q_{N}\left(q^{2 N}\right)=\prod_{k=N+1}^{2 N}\left(q^{k}-q^{-k}\right) .
$$

This amounts to (2.16) with $\delta=-$. For $\delta=+$ we use (2.14) to obtain

$$
K\left(x, i N a_{+}\right)=K\left(-x,-i N a_{+}\right)=K\left(x,-i N a_{+}\right) .
$$

To prove the last assertion of the theorem, we note that by virtue of $(2.13), K(x, p)$ satisfies the dual $\mathrm{A} \Delta \mathrm{E}$

$$
s_{-}\left(p+i N a_{+}\right) K\left(x, p-i a_{+}\right)+s_{-}\left(p-i N a_{+}\right) K\left(x, p+i a_{+}\right)=2 s_{-}(p) c_{-}(x) K(x, p) .
$$

Substituting $p=i N a_{+}$, this yields

$$
s_{-}\left(2 i N a_{+}\right) K\left(x, i(N-1) a_{+}\right)=2 s_{-}\left(i N a_{+}\right) c_{-}(x) K\left(x, i N a_{+}\right) .
$$

Assuming (2.17) from now on, we have $s_{-}\left(i k a_{+}\right) \neq 0$ for $k=1, \ldots, 2 N$. From (2.16) we then deduce that $K\left(x, i(N-1) a_{+}\right)$is a nonzero multiple of $c_{-}(x)$. Putting next $p=i(N-1) a_{+}$in the dual $\mathrm{A} \Delta \mathrm{E}$, we infer that $K\left(x, i(N-2) a_{+}\right)$is of the form $A c_{-}(x)^{2}+B$, with $A \neq 0$, etc. This yields (2.18) for $\delta=-$, and then the $\delta=+$ case follows from the evenness relation (2.14).

It should be noted that the self-duality property (2.13) entails that we have

$$
\hat{B}_{\delta} K_{N}(x, p)=2 c_{\delta}(x) K_{N}(x, p), \quad \delta=+,-,
$$

where $\hat{B}_{\delta}$ are the dual $\mathrm{A} \Delta \mathrm{Os}$

$$
\begin{gathered}
\hat{B}_{-} \equiv \frac{s_{-}\left(p+i N a_{+}\right)}{s_{-}(p)} \hat{T}_{i a_{+}}+(i \rightarrow-i), \\
\hat{B}_{+} \equiv(-)^{N} \hat{T}_{i a_{-}}+(i \rightarrow-i),
\end{gathered}
$$

with

$$
\left(\hat{T}_{\alpha} G\right)(p) \equiv G(p-\alpha), \quad \alpha \in \mathbb{C} .
$$

Thus $K_{N}(x, p)$ is a joint eigenfunction of four independent $\mathrm{A} \Delta \mathrm{O}$ s. [In fact, we already exploited (2.48) with $\delta=-$ in the above proof; cf. (2.46).]

We continue by detailing the relation between $K_{N}(x, p)$ and the function $\mathcal{H}_{N}(x, y)$ from Sec. II in I [cf. I(2.34)], specialized to the hyperbolic context. Consider the two-variable polynomial $S_{N}(r, t)(2.21)$. The coefficient of $t^{N}$ reads

$$
\sum_{k=0}^{N} c_{k N} r^{k}=(-)^{N} q^{-N(N+1) / 2} Q_{N}(r)=(-)^{N} q^{-N(N+1) / 2} \prod_{j=1}^{N}\left(1-q^{2 j} r\right)
$$

cf. (2.6). Similarly, the coefficient of $t^{0}$ reads 


$$
S(r, 0)=q^{N(N+1) / 2} Q_{0}(r)=q^{N(N+1) / 2} \prod_{j=1}^{N}\left(1-q^{-2 j} r\right) .
$$

Now we view $S(r, t)$ as a polynomial in $r$ with $t$-dependent coefficients, recalling $S(r, t)$ $=S(t, r)$. The coefficient of $r^{N}$ is therefore given by the rhs of (2.52) with $r \rightarrow t$. Assuming $t$ $\neq q^{-2 j}, j=1, \ldots, N$, from now on, it follows that $S(r, t)$ is of degree $N$ in $r$ and can be written as

$$
S(r, t)=q^{-N(N+1) / 2} \prod_{j=1}^{N}\left(1-q^{2 j} t\right)\left(\rho_{j}-r\right),
$$

where the roots $\rho_{j}$ depend on $q$ and $t$. Likewise, (2.53) entails

$$
S(0, t)=q^{N(N+1) / 2} \prod_{j=1}^{N}\left(1-q^{-2 j} t\right) .
$$

Hence, putting $r=0$ in (2.54), we deduce

$$
\prod_{j=1}^{N} \rho_{j}=q^{N(N+1)} \prod_{j=1}^{N} \frac{1-q^{-2 j} t}{1-q^{2 j} t} .
$$

In particular, none of the roots vanishes, provided $t \neq q^{2 j}, j=1, \ldots, N$. Moreover, from (2.53) we infer that the root $\rho_{j}$ may be chosen equal to $q^{2 j}$ for $t=0$.

We now rewrite $t$ as $e_{-}(-2 p)$, so that (2.56) becomes

$$
\prod_{j=1}^{N} \rho_{j}=\prod_{j=1}^{N} \frac{s_{-}\left(p+i j a_{+}\right)}{s_{-}\left(p-i j a_{+}\right)}
$$

Restricting attention to $\{\operatorname{Re} p>0\}$, we may introduce (continuous) functions $z_{j}(p)$ by requiring

$$
\rho_{j}=e_{-}\left(2 z_{j}\right), \quad z_{j}(p) \rightarrow i j a_{+}, \quad p \rightarrow \infty, \quad j=1, \ldots, N .
$$

Then a routine calculation [using (2.54)] yields

$$
e_{-}(N x+N p) S\left(e_{-}(-2 x), e_{-}(-2 p)\right)=2^{2 N} \prod_{j=1}^{N}\left[s_{-}\left(p+i j a_{+}\right) s_{-}\left(p-i j a_{+}\right)\right]^{1 / 2} s_{-}\left(x+z_{j}(p)\right) .
$$

It should be emphasized that the above holds true for all positive $a_{+}, a_{-}$. To establish contact with Sec. II in I, however, we should require (2.17); cf. I(2.25). Then it easily follows that the zeros $z_{j}(p)$ may be identified with the zeros $z_{j}(y)$ in loc. cit., with $p$ and $y$ related via (1.9), and that the relation to $\mathcal{H}_{N} \mathrm{I}(2.34)$, reads

$$
K_{N}(x, p)=\left(4 \pi / a_{-}\right)^{N} \prod_{j=1}^{N}\left[s_{-}\left(p+i j a_{+}\right) s_{-}\left(p-i j a_{+}\right)\right]^{1 / 2} \cdot \mathcal{H}_{N}\left(x, \pi p / a_{+} a_{-}\right) .
$$

Moreover, (2.17) entails nonconstancy in $p$ for all of the zeros $z_{j}(p)$. [Indeed, the coefficients $d_{l}$ $\mathrm{I}(2.46)$ in the asymptotics $\mathrm{I}(2.45)$ are nonzero.] We will show later on that $p$-independent zeros do occur when (2.17) is violated; equivalently, the polynomial $S_{N}(r, t)$ is not irreducible in that case.

It is a remarkable consequence of (the hyperbolic specialization of) Sec. II in I that all of the roots $\rho_{j}$ lie on the unit circle for $t \in(0, \epsilon)$ and $\epsilon$ small enough. For $N=1$ this remains true for all $t \in(0,1]$ and all $a_{+}, a_{-} \in(0, \infty)$; cf. (2.56). But already for $N=2,3$ and suitable $a_{+}, a_{-}$, the roots 
do not stay on the unit circle as $t$ goes to 1 . Hence, the functions $z_{j}(p)$ move off the imaginary axis as $p$ decreases from $\infty$ to 0 . This entails that the parameter $K$ is indispensable when one requires the $z_{j}$ to belong to $i(0, \infty)$-as we do in loc. cit.

To see the roots move off the unit circle for $N=2$, one need only use (2.9) to calculate

$$
S_{2}(r, 1)=\left(q^{3}-q-q^{-1}+q^{-3}\right)\left(1+C_{2} r+r^{2}\right), \quad C_{2} \equiv q^{2}+2+q^{-2} .
$$

Since we have $C_{2}=4 \cos ^{2}\left(\pi a_{+} / a_{-}\right)$, we get $C_{2} \in(2,4)$ for $a_{+} \in\left(0, a_{-} / 4\right)$ (say). Thus, the roots $\left[-C_{2} \pm\left(C_{2}^{2}-4\right)^{1 / 2}\right] / 2$ do not lie on the unit circle for $a_{+} / a_{-}<1 / 4$. Likewise, for $N=3$ one readily calculates from $(2.10)$,

$$
S_{3}(r, 1)=\left[q^{6}-q^{4}-q^{2}-\left(q \rightarrow q^{-1}\right)\right]\left[1+C_{3}\left(r+r^{2}\right)+r^{3}\right], \quad C_{3} \equiv q^{4}+2 q^{2}+3+2 q^{-2}+q^{-4} .
$$

For $q \rightarrow 1$ the roots therefore converge to those of the polynomial $(1+r)\left(1+8 r+r^{2}\right)$. From this it easily follows that for $a_{+} / a_{-}$small enough (at least) two roots move off the unit circle as $p \downarrow 0$.

Next, we reconsider the formula I(2.43) for the Casorati determinant $\mathrm{I}(2.41)$. In view of $(2.60)$ and (2.14) we may as well study

$$
D_{N}^{+}(x) \equiv K_{N}\left(x+i a_{+} / 2, p\right) K_{N}\left(x-i a_{+} / 2,-p\right)-(i \rightarrow-i) .
$$

Adapting the reasoning in loc. cit. to the present context, we obtain

$$
D_{N}^{+}(x)=\beta_{N}(p) \prod_{n=-N+1 / 2}^{N-1 / 2} s_{-}\left(x-i n a_{+}\right) .
$$

Indeed, the quotient of $D_{N}^{+}(x)$ and the product on the rhs is hyperbolic with period $i a_{-}$and pole-free. Since the quotient has finite limits for $\operatorname{Re} x \rightarrow \pm \infty$, it is $x$-independent.

Now the limit $\beta_{N}(p)$ of the quotient for $\operatorname{Re} x \rightarrow \infty$. (say) can be determined explicitly from (1.15); it reads

$$
\beta_{N}(p)=2^{2 N}\left[e_{-}(-p)-e_{-}(p)\right]\left(\sum_{l=0}^{N} c_{0 l} e_{-}((N-2 l) p)\right)(p \rightarrow-p) .
$$

Using $c_{0 l}=c_{l 0}$ and (2.4)-(2.6), this can be rewritten as

$$
\begin{aligned}
\beta_{N}(p) & =2^{2 N+1} s_{-}(-p) q^{N(N+1)} Q_{0}\left(e_{-}(-2 p)\right) Q_{0}\left(e_{-}(2 p)\right) \\
& =(-)^{N+1} 2^{4 N+1} \prod_{j=-N}^{N} s_{-}\left(p+i j a_{+}\right) .
\end{aligned}
$$

Recalling (2.60), we deduce that $\alpha_{N}$ in $\mathrm{I}(2.43)$ specializes to

$$
\alpha_{N}=(-)^{N+1} 2 \sinh \left(a_{+} y\right), \quad y=\pi p / a_{+} a_{-} .
$$

From (2.66) we read off that the Casorati determinant of the solutions $K_{N}(x, p)$ and $K_{N}(x,-p)$ to the $\mathrm{A} \Delta \mathrm{E}(2.12)$ vanishes identically iff $p$ equals $p_{j k} \equiv i j a_{+}+i k a_{-}$with $j=-N, \ldots, N$ and $k \in \mathbb{Z}$. For other $p$-values it then follows that the meromorphic quotient function $K_{N}(x, p) / K_{N}(x,-p)$ is not $i a_{+}$-periodic (cf. Appendix B in I). Moreover, from (2.18) we obtain

$$
K_{N}\left(x, p_{j k}\right)=e_{+}(-2 k x) K_{N}\left(x,-p_{j k}\right), \quad j=-N, \ldots, N, \quad k \in \mathbb{Z} .
$$

As should be the case, this yields an $i a_{+}$-periodic quotient $e_{+}(-2 k x)$ whenever the lhs does not vanish identically.

Consider next the Casorati determinant 


$$
D_{N}^{-}(x) \equiv K_{N}\left(x+i a_{-} / 2, p\right) K_{N}\left(x-i a_{-} / 2,-p\right)-(i \rightarrow-i),
$$

corresponding to the $\mathrm{A} \Delta \mathrm{E}$ (1.19). From (2.19)-(2.21), we obtain

$$
D_{N}^{-}(x)=-2 s_{+}(p) e_{-}(2 N x) S_{N}\left(q ;-e_{-}(-2 x), e_{-}(-2 p)\right) S_{N}\left(q ;-e_{-}(-2 x), e_{-}(2 p)\right) .
$$

Thus, $D_{N}^{-}(x)$ vanishes for $p=i j a_{+}, j \in \mathbb{Z}$, and for $p$ such that $K_{N}(x, p)=0$ identically, while for other $p$-values the quotient $K_{N}(x, p) / K_{N}(x,-p)$ is not $i a_{-}$-periodic. [Note that the functions $K_{N}\left(x, \pm i j a_{+}\right)$are manifestly either $i a_{-}$-periodic or $i a_{-}$-antiperiodic, depending on the parity of $j$.

Restricting attention to $\operatorname{Re} p \neq 0$, both $D_{N}^{+}(x)$ and $D_{N}^{-}(x)$ are nonzero. Then the reasoning in the proof of Theorem B.1 in I applies with various simplifications. It leads to the conclusion that for $a_{+} / a_{-} \notin \mathrm{Q}$ and $\operatorname{Re} p>0$ the joint eigenspace of the $\mathrm{A} \Delta \mathrm{O}$ pair $\left(B_{+}, B_{-}\right)$corresponding to eigenvalues $\left(2 c_{+}(p), 2 c_{-}(p)\right)$ is two-dimensional, and spanned by the functions $K_{N}( \pm x, p)$.

The result just arrived at amounts to a sharpening of Theorem B.1 in I for the hyperbolic integer $g$ case. It entails, in particular, that for $a_{+} / a_{-}$irrational the coefficients in $(2.21)$ must be proportional to $(2.5)$ whenever (2.1) holds true. Hence, the assertion in the sentence containing (2.1) easily follows.

It is of interest to point out a second, closely related corollary. Recall that we showed in the proof of Theorem II.1 that the system $d_{m n}=0$ with side conditions (2.25) and irrational $a_{+} / a_{-}$ has a unique solution $c_{k l}$ for arbitrary boundary coefficients $c_{0 n}$. We are now in the position to deduce that this solution does not vanish for all $l>N$ unless the boundary coefficients are proportional to $b_{n}(2.26)$ - a surprising fact that we are unable to establish directly.

We continue by deriving some features of the joint eigenfunction

$$
R_{N}(x, p) \equiv(-i)^{N+1}\left[K_{N}(x, p)-K_{N}(x,-p)\right]\left[P_{N}(x) P_{N}(p)\right]^{-1},
$$

of the $\mathrm{A} \Delta \mathrm{Os} A_{+}$and $A_{-}$. Notice that this definition entails, in particular,

$$
R_{0}(x, p)=\frac{\sin \left(\pi x p / a_{+} a_{-}\right)}{2 s_{-}(x) s_{-}(p)}
$$

cf. (2.19).

Theorem II.2: The function $R_{N}(x, p)$ satisfies the $A \Delta E$

$$
s_{-}\left(x-i(N+1) a_{+}\right) F\left(x-i a_{+}\right)+s_{-}\left(x+i(N+1) a_{+}\right) F\left(x+i a_{+}\right)=2 s_{-}(x) c_{-}(p) F(x) .
$$

It has the symmetry properties

$$
\begin{gathered}
R_{N}(x, p)=R_{N}(p, x), \\
R_{N}(x, p)=R_{N}(-x, p)=R_{N}(x,-p), \\
R_{N}(x, p)=\overline{R_{N}}(x, p), \quad x, p \in \mathbb{R} .
\end{gathered}
$$

Now assume

$$
a_{+} / a_{-} \notin \mathbb{Q}
$$

Then one has

$$
R_{N}\left(x, \pm i(N+1) a_{+}\right)=\prod_{k=N+1}^{2 N+1}\left[2 \sin \left(\pi k a_{+} / a_{-}\right)\right]^{-1}
$$


Moreover, one has

$$
R_{N}\left(x, i \delta(N+1+l) a_{+}\right)=G_{l}^{(N)}\left(c_{-}(x)\right), \quad l \in \mathbb{N}, \quad \delta=+,-,
$$

where $G_{l}^{(N)}(u)$ is a polynomial of degree $l$ and parity $(-)^{l}$ with real coefficients.

Proof: The features (2.73)-(2.76) readily follow from Theorem II.1. Combining (2.73) and (2.74) yields the dual $\mathrm{A} \Delta \mathrm{E}$

$$
s_{-}\left(p-i(N+1) a_{+}\right) R_{N}\left(x, p-i a_{+}\right)+(i \rightarrow-i)=2 s_{-}(p) c_{-}(x) R_{N}(x, p) .
$$

Substituting $p=i(N+1) a_{+}$, this reads

$$
s_{-}\left(2 i(N+1) a_{+}\right) R_{N}\left(x, i(N+2) a_{+}\right)=2 s_{-}\left(i(N+1) a_{+}\right) c_{-}(x) R_{N}\left(x, i(N+1) a_{+}\right) .
$$

Assuming (2.77) from now on, let us first take (2.78) for granted. Then (2.81) entails that $R_{N}\left(x, i(N+2) a_{+}\right)$is a nonzero real multiple of $c_{-}(x)$. Taking next $p=i(N+2) a_{+}$in $(2.80)$, we infer that $R_{N}\left(x, i(N+3) a_{+}\right)$is of the form $C c_{-}(x)^{2}+D$, with $C \in \mathbb{R}^{*}, D \in \mathbb{R}$. More generally, putting $p=i(N+l) a_{+}, l \in \mathbb{N}^{*}$, yields a three-term recurrence relation with coefficients in $i \mathbb{R}^{*}$, and so the last assertion of the theorem easily follows.

It remains to prove $(2.78)$. Due to $(2.71)$ this identity amounts to

$$
K_{N}\left(x,-i(N+1) a_{+}\right)-K_{N}\left(x, i(N+1) a_{+}\right)=(-)^{N} \prod_{j=-N_{N}}^{N}\left(q^{j} e_{-}(x)-q^{-j} e_{-}(-x)\right) \prod_{l=1}^{N}\left(q^{l}-q^{-l}\right)
$$

In view of (1.15) and (2.14), the lhs can be written as

$$
2 \sum_{k=0}^{N} L_{k} s_{-}((2 k+1) x)
$$

with

$$
\begin{aligned}
L_{k} & \equiv q^{N(N+1)} \sum_{l=0}^{N} c_{k l}^{(N)}(q) q^{-2 l(N+1)} \\
& =(-)^{k} q^{3 N(N+1) / 2} c_{k}^{(N)}\left(q^{-2}\right) \prod_{j \in I_{k}}\left(1-q^{-2 j} \cdot q^{-2(N+1)}\right), \quad k=0, \ldots, N .
\end{aligned}
$$

[Here we used $c_{k l}=c_{l k},(2.5)$ and (2.6).]

On the other hand, we have

$$
\begin{aligned}
\prod_{j=-N}^{N}\left(q^{j} e_{-}(x)-q^{-j} e_{-}(-x)\right) & =e_{-}((2 N+1) x) \prod_{j=-N}^{N}\left(1-q^{-2 j} e_{-}(-2 x)\right) \\
& =e_{-}((2 N+1) x) \sum_{m=0}^{2 N+1}(-)^{m} s_{m} e_{-}(-2 m x),
\end{aligned}
$$

where

$$
s_{m} \equiv \sum_{-N \leqslant j_{1}<\cdots<j_{m} \leqslant N} q^{-2\left(j_{1}+\cdots+j_{m}\right)}, \quad m=0, \ldots, 2 N+1 .
$$

Now one easily sees that $s_{2 N+1-m}=s_{m}$, so the rhs of (2.85) can be written as 


$$
2 \sum_{k=0}^{N} R_{k} s_{-}((2 k+1) x), \quad R_{k} \equiv(-)^{N-k} s_{N-k}, \quad k=0, \ldots, N .
$$

Comparing, we deduce that (2.82) is equivalent to the identities

$$
L_{k}=(-)^{N} R_{k} \prod_{l=1}^{N}\left(q^{l}-q^{-l}\right), \quad k=0, \ldots, N .
$$

We proceed by proving (2.88). First, we take $k=N$. Then (2.84) yields [cf. (2.2) and (2.4)]

$$
L_{N}=(-)^{N} q^{N(N+1) / 2} \prod_{l=1}^{N}\left(1-q^{-2 l}\right),
$$

whereas (2.87) and (2.86) imply $R_{N}=1$. Hence (2.88) holds true for $k=N$.

Next, we note that the recurrence relation (2.31) obtained in the proof of Theorem II.1 can be rewritten as

$$
\left(1-w^{-k}\right) c_{k}^{(N)}(w)=\left(w^{N-k+1}-1\right) c_{k-1}^{(N)}(w), \quad k=1, \ldots, N
$$

cf. (2.26) and (2.4). In view of (2.84) and (2.2), this entails

$$
\frac{L_{k}}{L_{k-1}}=\frac{1-w^{N-k+1}}{1-w^{-k}} \cdot \frac{1-w^{-N+k-1} \cdot w^{N+1}}{1-w^{k} \cdot w^{N+1}}=\frac{w^{k}-w^{N+1}}{w^{k+N+1}-1}, \quad k=1, \ldots, N .
$$

To conclude the proof of the theorem, it is therefore sufficient to show that the coefficients $R_{k}$ satisfy the recurrence relation $(2.91)$, too. Due to (2.87) this amounts to the recurrence

$$
\frac{s_{N-k}}{s_{N-k+1}}=\frac{w^{k}-w^{N+1}}{1-w^{k+N+1}}, \quad k=1, \ldots, N .
$$

To prove that (2.92) indeed holds, we observe that we may write (2.86) as

$$
s_{m}=w^{-m(N+1)} \sum_{1 \leqslant i_{1}<\cdots<i_{m} \leqslant 2 N+1} w^{i_{1}+\cdots+i_{m}}=w^{-m(N+1)} c_{m}^{(2 N+1)}(w) .
$$

Using (2.90) with $N \rightarrow 2 N+1$, we therefore have

$$
\frac{s_{m-1}}{s_{m}}=w^{N+1} \frac{1-w^{-m}}{w^{2 N-m+2}-1}, \quad m=1, \ldots, 2 N+1 .
$$

Putting $m=N-k+1$, this yields (2.92), completing the proof.

The polynomials $G_{l}^{(N)}(2.79)$ may be viewed as analytic continuations of $q_{t}^{2}$-Gegenbauer polynomials with $q_{t} \in(0,1)$ to $q$ on the unit circle; cf. (1.16). This will become clear from our study of the trigonometric setting, which we undertake in Sec. IV. Indeed, the results embodied in Theorems II. 1 and II.2 have trigonometric corollaries that can be obtained rather easily.

Our next and last theorem in this section has no bearing on the trigonometric case. Rather, it throws new light on the zero representation (2.59) and the restriction (2.17) corresponding to $\mathrm{I}(2.25)$. Moreover, the theorem plays a crucial role in Sec. III, where we handle the general hyperbolic case. It concerns the case of rational $a_{+} / a_{-}$, which we encode here as

$$
a_{+} / a_{-}=s / r, \quad s, r \in \mathbb{N}^{*}, \quad s, r \text { coprime. }
$$


Assuming (2.95), the restriction (2.17) is satisfied iff $N<r / 2$. Hence for $N<r / 2$ all of the zeros $z_{j}(p)$ on the rhs of (2.59) are $p$-dependent. [Recall the paragraph containing (2.60).] Now (2.59) was derived without restrictions on $a_{+}, a_{-}$, and $N$. In particular, it holds true for

$$
L=M+m r, \quad M \in \mathbb{N}, \quad M \leqslant r-1, \quad m \in \mathbb{N}^{*} .
$$

Our next result entails that in (2.59) we then have

$$
z_{j}(p)=i j a_{+}, \quad j=M+1, \ldots, L .
$$

That is, these zeros are $p$-independent and therefore equal to their limits for $p \rightarrow \infty$; $\mathrm{cf}$. (2.58). Moreover, for $r>1$ and $M \in[r / 2, r-1]$, one also has

$$
z_{j}(p)=i j a_{+}, \quad j=r-M, \ldots, M .
$$

The following theorem contains far more information than its easy corollaries just mentioned. Note, however, that the prefactors in the formulas (2.99) and (2.100) can be independently checked when one takes (2.97) and (2.98) for granted and uses (2.59).

Theorem II.3: Fix $a_{+}, a_{-}>0$ such that (2.95) holds true, and assume (2.96). Then one has

$$
K_{L}(x, p)=q^{L(L+1) / 2} q^{-M(M+1) / 2}\left[4 s_{-}(r x) s_{-}(r p)\right]^{m} K_{M}(x, p) .
$$

Next, assume $r>1$ and $M \in[r / 2, r-1]$. Then one has

$$
K_{M}(x, p)=\prod_{j=r}^{M}\left[4 q^{-j_{S}} s_{-}\left(x+i j a_{+}\right) s_{-}\left(p+i j a_{+}\right)\right] \cdot K_{N}(x, p), \quad N \equiv r-1-M .
$$

Proof: Since the variables

$$
q=\exp (i \pi s / r), \quad w=\exp (-2 i \pi s / r)
$$

are fixed, we may as well suppress them. Our starting point is the identity

$$
S_{N}(u, t)=q^{N(N+1) / 2} \sum_{n=0}^{N}(-t)^{n} c_{n}^{(N)} Q_{n}^{(N)}(u),
$$

which easily follows from the above definitions [cf. (2.21) and (2.2)-(2.6)]. It entails that (2.99) is equivalent to the relation

$$
\sum_{l=0}^{L}(-t)^{l} c_{l}^{(L)} Q_{l}^{(L)}(u)=\left(1-u^{r}\right)^{m}\left(1-t^{r}\right)^{m} \sum_{k=0}^{M}(-t)^{k} c_{k}^{(M)} Q_{k}^{(M)}(u) .
$$

We prove (2.103) in several steps. First, we note the identity

$$
\prod_{l=\rho+1}^{\rho+r}\left(1-w^{l} u\right)=1-u^{r}, \quad \rho \in \mathbb{Z} .
$$

Indeed, since $s$ and $r$ are coprime, the numbers $l s$, with $r$ consecutive integers $l$, are distinct $\bmod r$. Thus $(2.104)$ is a consequence of the identity

$$
\prod_{j=1}^{r}\left(1-\zeta^{j} u\right)=1-u^{r}, \quad \zeta \equiv \exp (-2 i \pi / r)
$$

whose proof is immediate. 
Second, we exploit (2.104) to relate $Q_{l}^{(L)}$ for $l$ of the form $k+j r$ with $k=0, \ldots, M$ and $j$ $=0, \ldots, m$ to $Q_{k}^{(M)}$. Specifically, from the definitions (2.6) and (2.2), we obtain

$$
\begin{aligned}
Q_{k+j r}^{(L)}(u) & =\prod_{l=-L}^{-L+k+j r-1}\left(1-w^{l} u\right) \cdot \prod_{l=k+j r+1}^{L}\left(1-w^{l} u\right) \\
& =\left(1-u^{r}\right)^{j} \prod_{l=-M}^{-M+k-1}\left(1-w^{l} u\right) \cdot\left(1-u^{r}\right)^{m-j} \prod_{l=k+1}^{M}\left(1-w^{l} u\right) \\
& =\left(1-u^{r}\right)^{m} Q_{k}^{(M)}(u), \quad k=0, \ldots, M, \quad j=0, \ldots, m .
\end{aligned}
$$

Third, we combine the special case $k, j=0$ of (2.106), which we rewrite as

$$
Q_{0}^{(L)}(u)=Q_{0}^{(M)}(u) \sum_{j=0}^{m}(-)^{j}\left(\begin{array}{c}
m \\
j
\end{array}\right) u^{j r},
$$

with the expansions

$$
Q_{0}^{(L)}(u)=\sum_{l=0}^{L} c_{l}^{(L)}(-u)^{l}, \quad Q_{0}^{(M)}(u)=\sum_{k=0}^{M} c_{k}^{(M)}(-u)^{k},
$$

which follow from (2.3)-(2.6). Since $Q_{0}^{(M)}(u)$ has degree $M<r$, this yields

$$
\begin{gathered}
c_{l}^{(L)}=0, \quad l=M+1, \ldots, r-1 \quad(\bmod r), \\
c_{k+j r}^{(L)}=(-)^{j r+j}\left(\begin{array}{c}
m \\
j
\end{array}\right) c_{k}^{(M)}, \quad k=0, \ldots, M, \quad j=0, \ldots, m .
\end{gathered}
$$

Fourth, we use (2.109), (2.110), and (2.106) to write

$$
\begin{aligned}
\sum_{l=0}^{L}(-t)^{l} c_{l}^{(L)} Q_{l}^{(L)}(u) & =\sum_{k=0}^{M} \sum_{j=0}^{m}(-t)^{k+j r} c_{k+j r}^{(L)} Q_{k+j r}^{(L)}(u) \\
& =\left(1-u^{r}\right)^{m} \sum_{k=0}^{M}(-t)^{k} c_{k}^{(M)} Q_{k}^{(M)}(u) \sum_{j=0}^{m}\left(-t^{r}\right)^{j}\left(\begin{array}{c}
m \\
j
\end{array}\right) \\
& =\left(1-u^{r}\right)^{m}\left(1-t^{r}\right)^{m} \sum_{k=0}^{M}(-t)^{k} c_{k}^{(M)} Q_{k}^{(M)}(u) .
\end{aligned}
$$

This equals (2.103), so (2.99) follows.

To prove $(2.100)$, we begin by noting that when we write

$$
Q_{j}^{(M)}(u)=P^{(M)}(u) R_{j}^{(M)}(u), \quad P^{(M)}(u) \equiv \prod_{k=r-M}^{M}\left(1-w^{k} u\right),
$$

then $R_{j}^{(M)}(u)$ is a polynomial of degree $N$. Of course, this is plain from (2.6) for $j=0, \ldots, N$, independently of the value of $w$. Since we have $w^{r}=1$ in the present case however, the remainder term $R_{j}^{(M)}(u)$ is still a polynomial for $j=r-M, \ldots, M$.

From (2.102) with $N \rightarrow M$ we now deduce that $S_{M}(u, t)$ is the product of $P^{(M)}(u)$ and a polynomial in $u$ and $t$. By self-duality (symmetry under $u \leftrightarrow t$ ) we then must have

$$
S_{M}(u, t)=P^{(M)}(u) P^{(M)}(t) P_{N}(u, t),
$$


where $P_{N}(u, t)$ is a polynomial of degree $N$ in $u$ and $t$, symmetric under the interchange of $u$ and $t$. Using (2.113) to rewrite the lhs of (2.100), it now follows from a straightforward calculation that (2.100) amounts to

$$
P_{N}(u, t)=\prod_{j=r-M}^{M} q^{j} \cdot S_{N}(u, t)
$$

Next, we observe that (2.114) holds true for $u=t=0$. [To check this, use $S_{K}(0,0)$ $=q^{K(K+1) / 2}$ and $P^{(M)}(0)=1$.] Thus, we need only show that the polynomials $P_{N}$ and $S_{N}$ are proportional. Switching back, this amounts to the quotient function

$$
Q_{N}(x, p) \equiv K_{M}(x, p) / \prod_{j=r_{-} M}^{M}\left[s_{-}\left(x+i j a_{+}\right) s_{-}\left(p+i j a_{+}\right)\right]
$$

being proportional to $K_{N}(x, p)$. We proceed by proving this, making suitable use of the first part of the proof of Theorem II.1.

First, we note that since $K_{M}(x, p)$ satisfies the $\mathrm{A} \Delta \mathrm{E}$ (2.12) with $N \rightarrow M$, we must have

$$
\begin{aligned}
& s_{-}\left(x+i M a_{+}\right) \prod_{j=r-M}^{M} s_{-}\left(x+i(j-1) a_{+}\right) \cdot Q_{N}\left(x-i a_{+}, p\right) \\
& +s_{-}\left(x-i M a_{+}\right) \prod_{j=r-M}^{M} s_{-}\left(x+i(j+1) a_{+}\right) \cdot Q_{N}\left(x-i a_{+}, p\right) \\
& =2 s_{-}(x) c_{-}(p) \prod_{j=r-M}^{M} s_{-}\left(x+i j a_{+}\right) \cdot Q_{N}(x, p) .
\end{aligned}
$$

When we now divide this by the product on the rhs and use the identity

$$
s_{-}\left(x-i M a_{+}\right) s_{-}\left(x+i(M+1) a_{+}\right)=s_{-}\left(x+i(r-M) a_{+}\right) s_{-}\left(x+i(M+1-r) a_{+}\right),
$$

then we obtain

$$
s_{-}\left(x+i N a_{+}\right) Q_{N}\left(x-i a_{+}, p\right)+(i \rightarrow-i)=2 s_{-}(x) c_{-}(p) Q_{N}(x, p) .
$$

Second, we recall that $K_{N}(x, p)$ also satisfies the $\mathrm{A} \Delta \mathrm{E}$ (2.118). Indeed, we used the general form (2.20)-(2.21) of $K_{N}(x, p)$ as an Ansatz to arrive at the system of equations $d_{m n}=0$ with side conditions (2.25), and then showed that the coefficients (2.5) solve this system. Now in view of (2.113) $Q_{N}(x, p)$ has the same general form as $K_{N}(x, p)$, except that the coefficients of the monomials in $P_{N}(u, t)$ are as yet unknown. We do know, however, that the coefficient matrix is symmetric.

Third, we reconsider the paragraph containing (2.26). Choosing $a_{+} / a_{-}$irrational guaranteed a unique solution for each set of boundary coefficients $b_{n}, n \in \mathbb{N}$. In the present case, however, $a_{+} / a_{-}$is rational, and we have a symmetric solution $\widetilde{c}_{k l}$ arising from $Q_{N}(x, p)$ on hand. The remaining problem, then, is to show that the latter coefficients equal the symmetric coefficients $c_{k l}^{(N)}$ occurring in $K_{N}(x, p)$, up to a common factor.

It is not hard to see that this is true. The key point is that we still have $w^{m} \neq 1$ for $m$ $=1, \ldots, N$. Hence a symmetric solution to the system is uniquely determined up to an overall factor. Indeed, starting from a given $c_{00}$, we can calculate successively $c_{10}, c_{20}, \ldots, c_{N 0}$, since $w^{m} \neq 1$. But then the boundary coefficients $c_{0 n}$ are determined by symmetry. Therefore, the remaining coefficients can be successively calculated (again because $w^{m} \neq 1$ ), entailing uniqueness. 


\section{THE GENERAL HYPERBOLIC CASE}

Just as in the special integer $g$ case studied in Sec. II, it is easy to adapt our results for the general elliptic case (cf. Sec. III in I) to the hyperbolic regime. But the results from Sec. II can actually be exploited to proceed considerably beyond the hyperbolic specialization of Sec. III in I. Indeed, we are going to obtain joint eigenfunctions for all parameters in the space $\mathcal{D}_{\text {hyp }}(1.7)$ and for all $p \in \mathbb{C}$. Moreover, for parameters in the subset $\mathcal{D}$ [defined by $\mathrm{I}(3.33)-\mathrm{I}(3.35)$ ], the representation derived below is far more explicit than the zero representation $\mathrm{I}(3.39)$.

We have occasion to make extensive use of the results obtained in Sec. II. To prevent ambiguous notation, the function $K_{N}(x, p)(2.20)$ is henceforth denoted by $K_{N}\left(a_{+}, a_{-} ; x, p\right)$. We also need two "q-variables," viz.,

$$
q_{+} \equiv \exp \left(i \pi a_{+} / a_{-}\right), \quad q_{-} \equiv \exp \left(i \pi a_{-} / a_{+}\right) .
$$

Thus $q$ (1.16) is, from now on, denoted by $q_{+}$.

To ease the exposition, we restrict attention to $b$-values of the form (1.20) until further notice, and, accordingly, study the auxiliary $\mathrm{A} \Delta \mathrm{O} B_{\delta}(1.21)$. We now claim that the functions

$$
K_{N_{+}, N_{-}}\left(a_{+}, a_{-} ; x, p\right) \equiv \exp \left(i \pi x p / a_{+} a_{-}\right) \prod_{\delta=+,-} e_{\delta}\left(N_{\delta}[x+p]\right) S_{N_{\delta}}\left(q_{\delta} ; e_{-\delta}(-2 x), e_{-\delta}(-2 p)\right)
$$

are joint $B_{\delta}$-eigenfunctions with eigenvalues $2 c_{\delta}(p)$. Given Theorem II.1, this is quite easily verified: For $B_{-}$we can use the identity

$$
K_{N_{+}, N_{-}}\left(a_{+}, a_{-} ; x, p\right)=K_{N_{+}}\left(a_{+}, a_{-} ; x, p\right) e_{+}\left(N_{-}[x+p]\right) S_{N_{-}}\left(q_{-} ; e_{+}(-2 x), e_{+}(-2 p)\right),
$$

whereas for $B_{+}$we can use

$$
K_{N_{+}, N_{-}}\left(a_{+}, a_{-} ; x, p\right)=K_{N_{-}}\left(a_{-}, a_{+} ; x, p\right) e_{-}\left(N_{+}[x+p]\right) S_{N_{+}}\left(q_{+} ; e_{-}(-2 x), e_{-}(-2 p)\right) .
$$

The joint eigenfunction property just demonstrated holds true for arbitrary $a_{+}, a_{-}>0$. Restricting $a_{+}$and $a_{-}$by I(3.34) and I(3.35), respectively, we also obtain a joint $B_{\delta}$-eigenfunction $\mathcal{H}(x, y) \mathrm{I}(3.39)$ in a quite different guise. Again, from Sec. II the connection between the two representations is easily established: One has

$$
K_{N_{+}, N_{-}}\left(a_{+}, a_{-} ; x, p\right)=\prod_{\delta=+,-}\left(\prod_{ \pm j=1}^{N_{\delta}} \frac{4 \pi}{a_{-\delta}} \sinh \frac{\pi}{a_{-\delta}}\left(p+i j a_{\delta}\right)\right)^{1 / 2} \cdot \mathcal{H}\left(x, \pi p / a_{+} a_{-}\right)
$$

[To see this, note first of all that $\mathrm{I}(3.17)$ becomes $y=u$ in the hyperbolic case. Canceling the plane wave $K_{0}(x, p)$ in the relation (2.60), the resulting formula readily yields (3.5).]

Next, we observe that the Casorati determinants

$$
D_{N_{+}, N_{-}}^{\delta}(x) \equiv K_{N_{+}, N_{-}}\left(a_{+}, a_{-} ; x+i a_{\delta} / 2, p\right) K_{N_{+}, N_{-}}\left(a_{+}, a_{-} ; x-i a_{\delta} / 2,-p\right)-(i \rightarrow-i)
$$

can be explicitly determined from (2.63)-(2.66) by using (3.3)/(3.4) for $\delta=+1-$. This yields

$$
\begin{aligned}
D_{N_{+}, N_{-}}^{\delta}(x)= & (-)^{N_{\delta}+1} \prod_{k=-N_{\delta}}^{N_{\delta}} 2 s_{-\delta}\left(p-i k a_{\delta}\right) \cdot \prod_{l=-N_{\delta}^{+1 / 2}}^{N_{\delta}-1 / 2} 2 s_{-\delta}\left(x-i l a_{\delta}\right) \\
& \cdot e_{\delta}\left(2 N_{-\delta} x\right) \prod_{\alpha=+,-} S_{N_{-}}\left(q-\delta ;-e_{\delta}(-2 x), e_{\delta}(2 \alpha p)\right), \quad \delta=+,-
\end{aligned}
$$


Furthermore, it follows as before that the determinants do not vanish identically for $\operatorname{Re} p \neq 0$. Adapting Theorem B.1 in I, we infer that for $a_{+} / a_{-}$irrational and $\operatorname{Re} p>0$ the functions $K_{N_{+}, N_{-}}\left(a_{+}, a_{-} ; x, \pm p\right)$ form a basis for the joint eigenspace of $B_{+}$and $B_{-}$corresponding to eigenvalues $2 c_{+}(p)$ and $2 c_{-}(p)$, respectively.

Next, we recall from Sec. III in I that for points $\left(a_{+}, a_{-}, b\right) \in \mathcal{D}$ there is at most one way to write $b$ as $\left(N_{+}+1\right) a_{+}-N_{-} a_{-}$with $N_{+}, N_{-} \in \mathbb{N}$ [cf. the paragraph containing I(3.29)]. Returning to the general case $a_{+}, a_{-}>0$, this is no longer true, of course. In particular, let us choose

$$
\frac{a_{+}}{a_{-}}=\frac{n_{-}}{n_{+}}, \quad n_{+}, n_{-} \in \mathbb{N}^{*}, \quad n_{+}, n_{-} \text {coprime. }
$$

Then we may rewrite $(1.20)$ as

$$
b_{+-}=\left(N_{+}+1+m n_{+}\right) a_{+}-\left(N_{-}+m n_{-}\right) a_{-},
$$

where $m$ is an arbitrary integer.

Choosing $m \in \mathbb{N}$, we now deduce from the identity (2.99) that we have

$$
\begin{gathered}
K_{N_{+}+m n_{+}, N_{-}+m n_{-}}\left(a_{+}, a_{-} ; x, p\right)=\eta_{N_{+}, N_{-}}(m) \prod_{\delta=+,-}\left[4 s_{-\delta}\left(n_{\delta} x\right) s_{-\delta}\left(n_{\delta} p\right)\right]^{m} \\
\cdot K_{N_{+}, N_{-}}\left(a_{+}, a_{-} ; x, p\right),
\end{gathered}
$$

where $\eta \in\{ \pm 1, \pm i\}$ is given by

$$
\eta_{N_{+}, N_{-}}(m) \equiv(-)^{m N_{+} n_{-}+m N_{-} n_{+}+m^{2} n_{+} n_{-} \cdot i^{m n_{-}+m n_{+}} .}
$$

All of the functions on the rhs of (3.10) are manifestly independent, so we wind up with an infinity of joint eigenfunctions for the same $b$-value!

We proceed by connecting the ambiguity just uncovered to the interpolation question discussed below I(4.8). As we have seen there, we get distinct weight functions $\hat{w}(x)$ for distinct $m \in \mathbb{N}$; cf. I(4.6). Moreover, in the elliptic case the A $\Delta$ Os $B_{\delta}$ also depend on the choice of $m$. But as we have already detailed in the Introduction, the hyperbolic counterparts (1.21) do admit the continuous interpolation $B_{\delta}(b)$ (1.22). [A caveat is in order at this point: For $b$ of the form $\left(N_{-}+1\right) a_{-}-N_{+} a_{+}$one would need a different interpolation. Specifically, one must take $b$ $\rightarrow a_{+}+a_{-}-b$ on the rhs of (1.22) in that case.]

This fact leads to a remarkable conclusion of a general character that we wish to emphasize before we discard the auxiliary $\mathrm{A} \Delta \mathrm{Os} B_{\delta}$ in favor of the $\mathrm{A} \Delta \mathrm{Os} A_{\delta}(b)(1.1)$ and $H_{\delta}(b)(1.5)$, which are defined for arbitrary real $b$ to begin with. Indeed, since the functions (3.10) are independent for different $m \in \mathbb{N}$, we may deduce that the commuting A $\Delta \mathrm{O}$ pair $B_{\delta}(b)(1.22)$ does not admit joint eigenfunctions depending continuously on the parameters, already for parameters $a_{+}, a_{-}>0$ and $b$ of the form (1.20). [In virtue of the specialization of Theorem B.1 in I, the ambiguity (3.10) is inescapable.]

This shows by example that the existence of interpolations cannot follow from general arguments. It is all the more remarkable that for the $\mathrm{A} \Delta \mathrm{OS} H_{\delta}(b)(1.5)$ [and hence for $A_{\delta}(b)(1.1)$, too] the interpolation ambiguity disappears: The ambiguity in the joint $B_{\delta}$-eigenfunctions is canceled by the ambiguity in the auxiliary weight function $\hat{w}(x)$.

To detail this, we first introduce the renormalized weight function

$$
\hat{w}_{N_{+}, N_{-}}\left(a_{+}, a_{-} ; x\right) \equiv 1 / \prod_{\delta=+,-} \prod_{ \pm j=1}^{N_{\delta}}\left[2 \sinh \frac{\pi}{a_{-\delta}}\left(x+i j a_{\delta}\right)\right] .
$$

[It differs from the hyperbolic specialization of $\hat{w}(x) \mathrm{I}(4.6)$ by a multiplicative constant.] With the rationality assumption (3.8) in effect, it satisfies 


$$
\hat{w}_{N_{+}+m n_{+}, N_{-}+m n_{-}}\left(a_{+}, a_{-} ; x\right)=\prod_{\delta=+,-}\left[2 s_{-\delta}\left(n_{\delta} x\right)\right]^{-2 m} \cdot \hat{w}_{N_{+}, N_{-}}\left(a_{+}, a_{-} ; x\right) .
$$

[Indeed, this comes down to the identity (2.104).]

Consider now the functions

$$
\begin{aligned}
& F_{N_{+}, N_{-}}\left(a_{+}, a_{-} ; x, p\right) \\
& \quad \equiv \phi_{N_{+}, N_{-}}\left[\hat{w}_{N_{+}, N_{-}}\left(a_{+}, a_{-} ; x\right) \hat{w}_{N_{+}, N_{-}}\left(a_{+}, a_{-} ; p\right)\right]^{1 / 2} K_{N_{+}, N_{-}}\left(a_{+}, a_{-} ; x, p\right),
\end{aligned}
$$

where $\phi$ is the phase

$$
\phi_{N_{+}, N_{-}} \equiv(-i)^{2 N_{+} N_{-}+N_{+}+N_{-}+1}
$$

By construction, they are joint eigenfunctions of the A $\Delta \mathrm{Os} H_{\delta}\left(\left(N_{+}+1\right) a_{+}-N_{-} a_{-}\right)$with eigenvalues $2 c_{\delta}(p)$. The phase satisfies

$$
\phi_{N_{+}+m n_{+}, N_{-}+m n_{-}}=\phi_{N_{+}, N_{-}} \bar{\eta}_{N_{+}, N_{-}}(m),
$$

so with (3.8) in force one deduces the equality

$$
F_{N_{+}+m n_{+}, N_{-}+m n_{-}}\left(a_{+}, a_{-} ; x, p\right)=F_{N_{+}, N_{-}}\left(a_{+}, a_{-} ; x, p\right), \quad m \in \mathbb{N} .
$$

Hence the ambiguities cancel out, as announced.

It should be noted that the definition (3.14) preserves the symmetry under $x \leftrightarrow p$. Moreover, it entails that we have

$$
F_{N_{+}, N_{-}}\left(a_{+}, a_{-} ; x, p\right)=\mathcal{F}\left(x, \pi p / a_{+} a_{-}\right),
$$

where $\mathcal{F}(x, y)$ is the hyperbolic specialization of I(3.31). Indeed, equality up to phase follows via (3.5), so we need only verify that the phase of the normalization constant $\mathcal{N}$ in the $c$-function I(1.25) equals $\phi_{N_{+}, N_{-}}$(3.15). Now from Proposition III. 8 in Ref. 2 we easily calculate

$$
c\left(a_{+}, a_{-},\left(N_{+}+1\right) a_{+}-N_{-} a_{-} ; x\right)=\phi_{N_{+}, N_{-}} \frac{\Pi_{k=1}^{N_{-}} 2 s_{+}\left(x+i k a_{-}\right)}{\Pi_{j=0}^{N_{+}} 2 s_{-}\left(x-i j a_{+}\right)} .
$$

Hence the phase $\phi(\mathcal{N})$ in I(3.31) indeed equals (3.15) in the hyperbolic case. (In fact, it is not hard to see that this is still true in the elliptic case.)

Thus far we have assumed $b$-values of the form (1.20). Let us next assume $b$-values of the form

$$
b_{-+}=-N_{+} a_{+}+\left(N_{-}+1\right) a_{-}, \quad N_{+}, N_{-} \in \mathbb{N} .
$$

Rewriting $H_{\delta}(b)(1.5)$ as

$$
H_{\delta}(b)=\left(\frac{s_{\delta}(x-i b) s_{\delta}\left(x+i b-i a_{-\delta}\right)}{s_{\delta}(x) s_{\delta}\left(x-i a_{-\delta}\right)}\right)^{1 / 2} T_{i a_{-\delta}}+(i \rightarrow-i), \quad \delta=+,-,
$$

we read off the symmetry property

$$
H_{\delta}(b)=H_{\delta}\left(a_{+}+a_{-}-b\right) .
$$


Thus, we may and will choose as joint eigenfunctions of $H_{+}\left(b_{-+}\right)$and $H_{-}\left(b_{-+}\right)$the functions $F_{N_{+}, N_{-}}\left(a_{+}, a_{-} ; x, p\right)$ just defined.

More generally, we obtain the same $\mathrm{A} \Delta \mathrm{O}$ pair $H_{\delta}(b)$ for the four $b$-values in the set

$$
B_{N_{+}, N_{-}} \equiv\left\{b_{+-}, b_{-+}, b_{--}, b_{++}\right\},
$$

where we use the notation (1.20), (3.20), and

$$
b_{--} \equiv-N_{+} a_{+}-N_{-} a_{-}, \quad b_{++} \equiv\left(N_{+}+1\right) a_{+}+\left(N_{-}+1\right) a_{-} .
$$

[Once more, this can be read off from (3.21).] But then we have

$$
H_{\delta}(b) F_{N_{+}, N_{-}}\left(a_{+}, a_{-} ; x, p\right)=2 c_{\delta}(p) F_{N_{+}, N_{-}}\left(a_{+}, a_{-} ; x, p\right), \quad b \in B_{N_{+}, N_{-}} .
$$

Hence, we have now constructed joint eigenfunctions for all parameters in $\mathcal{D}_{\text {hyp }}(1.7)$, as advertised in the Introduction.

But more can and should be said. In particular, for the rational case (3.8) we have shown the absence of ambiguity for positive $m$ in (1.20), but, of course, we can just as well choose $m$ equal to a negative integer. As long as $N_{+}+m n_{+}$and $N_{-}+m n_{-}$are non-negative, it is clear one still obtains (3.17). But when one of these integers becomes negative, the state of affairs is quite unclear at this stage. The next theorem supplies, in particular, the information that will enable us to unambiguously define a joint $H_{\delta}$-eigenfunction $F\left(a_{+}, a_{-}, b ; x, p\right)$ for all $\left(a_{+}, a_{-}, b\right)$ in $\mathcal{D}_{\text {hyp }}$. But it also yields additional information about the rational case (3.8) that is of interest in itself.

Theorem III.1: The function $F_{N_{+}, N_{-}}\left(a_{+}, a_{-} ; x, p\right)$ (3.14) satisfies

$$
F_{N_{+}, N_{-}}\left(a_{+}, a_{-} ; x, p\right)=F_{N_{-}, N_{+}}\left(a_{-}, a_{+} ; x, p\right) .
$$

Now assume (3.8). Fixing $N_{+}, N_{-} \in \mathbb{N}$, one has

$$
\begin{aligned}
& F_{N_{+}+m_{+} n_{+}, N_{-}+m_{-} n_{-}}\left(a_{+}, a_{-} ; x, p\right)=\zeta_{N_{+}, N_{-}}\left(m_{+}, m_{-}\right) F_{N_{+}, N_{-}}\left(a_{+}, a_{-} ; x, p\right), \\
& \zeta_{N_{+}, N_{-}}\left(m_{+}, m_{-}\right) \equiv(-)^{\left(m_{+}-m_{-}\right)\left(N_{-} n_{+}-N_{+} n_{-}\right)} \cdot i^{\left(m_{+}-m_{-}\right)\left[n_{-}-n_{+}+\left(m_{+}-m_{-}\right) n_{+} n_{-}\right]},
\end{aligned}
$$

where $m_{+}$and $m_{-}$are integers such that $N_{\delta}+m_{\delta} n_{\delta} \geqslant 0, \delta=+,-$. Moreover, choosing $N_{+}$ $\in\left[0, n_{+} / 2\right)$, one has

$$
\begin{gathered}
F_{M_{+}, N_{-}}\left(a_{+}, a_{-} ; x, p\right)=\xi_{N_{+}, N_{-}} F_{N_{+}, N_{-}}\left(a_{+}, a_{-} ; x, p\right), \quad M_{+} \equiv n_{+}-1-N_{+}, \\
\xi_{N_{+}, N_{-}} \equiv(-)^{\left(n_{+}-1\right) N_{-}+\left(n_{-}-1\right) N_{+} \cdot i^{n_{+} n_{-}-n_{-}-n_{+}+1} .}
\end{gathered}
$$

Proof: The symmetry property (3.26) can be read off from the definitions (3.14), (3.15), (3.12), and (3.2). To prove (3.27), we first note that (3.13) generalizes as

$$
\hat{w}_{N_{+}+m_{+} n_{+}, N_{-}+m_{-} n_{-}}\left(a_{+}, a_{-} ; x\right)=\prod_{\delta=+,-}\left[2 s_{-\delta}\left(n_{\delta} x\right)\right]^{-2 m_{\delta}} \hat{w}_{N_{+}, N_{-}}\left(a_{+}, a_{-} ; x\right) .
$$

Second, we can use the identity (2.99) once more to generalize (3.10). A straightforward calculation yields

$$
\begin{aligned}
& K_{N_{+}+m_{+} n_{+}, N_{-}+m_{-} n_{-}}\left(a_{+}, a_{-} ; x, p\right) \\
& \quad=\eta_{N_{+}, N_{-}}\left(m_{+}, m_{-}\right) \prod_{\delta=+,-}\left[4 s_{-\delta}\left(n_{\delta} x\right) s_{-\delta}\left(n_{\delta} p\right)\right]^{m_{\delta}} \cdot K_{N_{+}, N_{-}}\left(a_{+}, a_{-} ; x, p\right),
\end{aligned}
$$




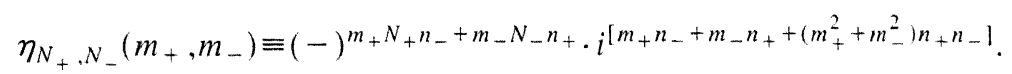

Third, (3.16) generalizes to

$$
\phi_{N_{+}+m_{+} n_{+}, N_{-}+m_{-} n_{-}}=\phi_{N_{+}, N_{-}} \zeta_{N_{+}, N_{-}}\left(m_{+}, m_{-}\right) \bar{\eta}_{N_{+}, N_{-}}\left(m_{+}, m_{-}\right) .
$$

Combining these relations, we obtain (3.27).

In order to prove (3.29), we note first

$$
\hat{w}_{M_{+}, N_{-}}\left(a_{+}, a_{-} ; x\right)=\prod_{ \pm j=N_{+}+1}^{n_{+}-1-N_{+}}\left[2 s_{-}\left(x+i j a_{+}\right)\right]^{-1} \cdot \hat{w}_{N_{+}, N_{-}}\left(a_{+}, a_{-} ; x\right) .
$$

Second, we exploit (2.100) to write

$$
K_{M_{+}, N_{-}}\left(a_{+}, a_{-} ; x, p\right)=\prod_{j=N_{+}+1}^{n_{+}-1-N_{+}}\left[4 q_{+}^{-j} s_{-}\left(x+i j a_{+}\right) s_{-}\left(p+i j a_{+}\right)\right] \cdot K_{N_{+}, N_{-}}\left(a_{+}, a_{-} ; x, p\right) \text {. }
$$

Consider now the function

$$
Q(x) \equiv \frac{\Pi_{j=N_{+}+1}^{n_{+}-1-N_{+}} q_{+}^{-j} s_{-}\left(x+i j a_{+}\right)}{\left[\prod_{ \pm j=N_{+}+1}^{n_{+}-1-N_{+}} s_{-}\left(x+i j a_{+}\right)\right]^{1 / 2}}
$$

From the identity

$$
s_{-}\left(x-i j a_{+}\right)=(-)^{n_{-}} s_{-}\left(x+i\left(n_{+}-j\right) a_{+}\right)
$$

we deduce that $Q(x)$ equals a phase, so taking $x \rightarrow \infty$ we obtain $Q(x)=1$. Hence (3.14) yields

$$
F_{M_{+}, N_{-}}\left(a_{+}, a_{-} ; x, p\right)=\phi_{M_{+}, N_{-}} \bar{\phi}_{N_{+}, N_{-}}^{n_{+}} \prod_{j=N_{+}+1}^{-N_{+}} q_{+}^{j} \cdot F_{N_{+}, N_{-}}\left(a_{+}, a_{-} ; x, p\right) \text {. }
$$

Calculating the phase yields the rhs of (3.30), so (3.29) follows.

Still assuming (3.8), this theorem shows that the vector space spanned by the functions $F_{M_{+}, M_{-}}, M_{+}, M_{-} \in \mathbb{N}$, is finite-dimensional: It is already spanned by the functions $F_{N_{+}, N_{-}}$with $N_{\delta} \in\left[0 . n_{\delta} / 2\right), \delta=+,-$. Indeed, all of the former functions are phase multiples of the latter, as follows by combining (3.27), (3.29), and (3.26). This fact is in accordance with (but not implied by) the relation

$$
H_{\delta}\left(b+m n_{+} a_{+}\right)=H_{\delta}\left(b+m n_{-} a_{-}\right)=H_{\delta}(b), \quad m \in Z, \quad \delta=+,-,
$$

whose validity is clear from (3.21).

More importantly, the theorem enables us to dispose of the $m \in Z$ ambiguity in (3.9) and its $b_{-+}$-analog. Specifically, taking $N_{+}, N_{-} \in \mathbb{N}$, we set [recall (1.20), (3.20), and (3.24)]

$$
\begin{aligned}
& F\left(a_{+}, a_{-}, b_{+-} ; x, p\right) \equiv F_{N_{+}, N_{-}}\left(a_{+}, a_{-} ; x, p\right), \\
& F\left(a_{+}, a_{-}, b_{-+} ; x, p\right) \equiv F_{N_{+}, N_{-}}\left(a_{+}, a_{-} ; x, p\right), \\
& F\left(a_{+}, a_{-}, b_{--} ; x, p\right) \equiv \widetilde{F}_{N_{+}, N_{-}}\left(a_{+}, a_{-} ; x, p\right), \\
& F\left(a_{+}, a_{-}, b_{++} ; x, p\right) \equiv \widetilde{F}_{N_{+}, N_{-}}\left(a_{+}, a_{-} ; x, p\right),
\end{aligned}
$$


where

$$
\begin{gathered}
\widetilde{F}_{N_{+}, N_{-}}\left(a_{+}, a_{-} ; x, p\right) \equiv \chi_{N_{+}, N_{-}} F_{N_{+}, N_{-}}\left(a_{+}, a_{-} ; x, p\right), \\
\chi_{N_{+}, N_{-}} \equiv(-)^{N_{+}+N_{-} \cdot i .}
\end{gathered}
$$

Of course, we are free to do so for $a_{+} / a_{-} \notin 0$, since then all $b$-values $k a_{+}+l a_{-}, k, l \in \mathbb{Z}$, are distinct. But our task is now to show that for the rational case (3.8) the function $F\left(a_{+}, a_{-}, b ; x, p\right)$ is still well defined.

Now we have already seen that (3.41) by itself is a legitimate definition; cf. (3.17). In view of the symmetry property (3.26), this is true for (3.42) as well. For (3.43) and (3.44) to be well defined by themselves, we should have

$$
\widetilde{F}_{N_{+}+m n_{+}, N_{-}}\left(a_{+}, a_{-} ; x, p\right)=\widetilde{F}_{N_{+}, N_{-}+m n_{-}}\left(a_{+}, a_{-} ; x, p\right), \quad m \in \mathbb{X} .
$$

Recalling (3.27), we see that this amounts to

$$
\zeta_{N_{+}, N_{-}}(m, 0) \chi_{N_{+}+m n_{+}, N_{-}}=\zeta_{N_{+}, N_{-}}(0 . m) \chi_{N_{+}, N_{-}+m n_{-}},
$$

which is easily verified. To prove the compatibility of (3.41) and (3.43), we need to show that when $M_{+} \in\left[n_{+} / 2, n_{+}-1\right]$, then we have

$$
F_{M_{+}, N_{-}+n_{-}}\left(a_{+}, a_{-} ; x, p\right)=\widetilde{F}_{N_{+}, N_{-}}\left(a_{+}, a_{-} ; x, p\right), \quad N_{+} \equiv n_{+}-1-M_{+} .
$$

Combining (3.27) and (3.29), we deduce that this amounts to the relation

$$
\zeta_{M_{+}, N_{-}}(0,1) \xi_{N_{+}, N_{-}}=\chi_{N_{+}, N_{-}}, \quad M_{+}=n_{+}-1-N_{+} .
$$

The phase $\chi_{N_{+}, N_{-}}$obeys this relation (indeed, it is defined such that it does), so (3.49) follows. The remaining compatibilities can now be handled by using (3.26). Thus, the function $F(\Xi ; x, p)$ is well defined for all parameters $\Xi=\left(a_{+}, a_{-}, b\right)$ in $\mathcal{D}_{\text {hyp }}(1.7)$.

We proceed by summarizing some salient features of the function $F(\Xi ; x, p)$.

Theorem III.2: For all $\Xi \in \mathcal{D}_{\text {hyp }}$ the definition (3.41)-(3.44) gives rise to a well-defined, generically two-valued, analytic function $F(\Xi ; x, p)$ with a meromorphic square. It satisfies

$$
H_{\delta} F(\Xi ; x, p)=2 c_{\delta}(p) F(\Xi ; x, p), \quad \delta=+,-,
$$

and has parameter and variable symmetries

$$
\begin{gathered}
F\left(a_{+}, a_{-}, b ; x, p\right)=F\left(a_{+}, a_{-}, a_{+}+a_{-}-b ; x, p\right), \\
F\left(a_{+}, a_{-}, b ; x, p\right)=F\left(a_{-}, a_{+}, b ; x, p\right), \\
F(\Xi ; x, p)=F(\Xi ; p, x), \\
F(\Xi ; x, p)=F(\Xi ;-x,-p) .
\end{gathered}
$$

Now denote by $F_{r}$ the function defined for $x, p>0$ by taking positive square roots in (3.14). For $a_{+} l a_{-} \notin\left(\right.$ ) this function has a real-analytic extension $F_{r}$ to $x, p \in \mathbb{R}$, which satisfies

$$
\begin{gathered}
F_{r}\left(a_{+}, a_{-}, b ; x, p\right)=-\bar{F}_{r}\left(a_{+}, a_{-}, b ;-x, p\right), \quad b=b_{+-}, b_{-+}, \\
F_{r}\left(a_{+}, a_{-}, b ; x, p\right)=\overline{F_{r}}\left(a_{+}, a_{-}, b ;-x, p\right), \quad b=b_{--}, b_{++} .
\end{gathered}
$$


Proof: It remains to prove (3.54)-(3.57). By virtue of (3.3) and (3.4), the holomorphic function (3.2) satisfies

$$
\begin{gathered}
K_{N_{+}, N_{-}}\left(a_{+}, a_{-} ; x, p\right)=K_{N_{+}, N_{-}}\left(a_{+}, a_{-} ; p, x\right), \\
K_{N_{+}, N_{-}}\left(a_{+}, a_{-} ; x, p\right)=K_{N_{+}, N_{-}}\left(a_{+}, a_{-} ;-x,-p\right), \\
K_{N_{+}, N_{-}}\left(a_{+}, a_{-} ; x, p\right)=(-)^{N_{+}+N_{-}} \bar{K}_{N_{+}, N_{-}}\left(a_{+}, a_{-} ;-x, p\right), \quad x, p \in \mathbb{R} .
\end{gathered}
$$

[Recall (2.13)-(2.15).] In view of (3.14), this entails

$$
\begin{gathered}
F_{N_{+}, N_{-}}\left(a_{+}, a_{-} ; x, p\right)=F_{N_{+}, N_{-}}\left(a_{+}, a_{-} ; p, x\right), \\
F_{N_{+}, N_{-}}\left(a_{+}, a_{-} ; x, p\right)=F_{N_{+}, N_{-}}\left(a_{+}, a_{-} ;-x,-p\right),
\end{gathered}
$$

so (3.54) and (3.55) follow. For $a_{+} / a_{-}$irrational, the auxiliary weight function (3.12) has a real-analytic, positive, and even restriction to $\mathbb{R}$, so (3.56) and (3.57) follow from (3.60) and the phase definitions (3.15) and (3.46).

Of course, for $a_{+} / a_{-}$rational, the restriction $F_{r}$ is still real-analytic for $x, p>0$. But in that case the weight function (3.12) may have poles at the origin, so that ambiguities can arise for $x$ $<0$. (Taking a real-analytic restriction to $\mathbb{R}$ and taking parameter limits need not commute; we mention the function $x \mapsto\left(x^{2}+\epsilon^{2}\right)^{-1 / 2}$ to exemplify this difficulty.)

Such square-root subtleties are not present for the meromorphic joint $A_{\delta}$-eigenfunction

$$
M(\Xi ; x, p) \equiv[w(\Xi ; x) w(\Xi ; p)]^{-1 / 2} F(\Xi ; x, p), \quad \Xi \in \mathcal{D}_{\mathrm{hyp}},
$$

which we study next. From Ref. 2 Eq. (5.21), we have

$$
\begin{aligned}
& w\left(a_{+}, a_{-}, k_{+} a_{+}+k_{-} a_{-} ; x\right) \\
& \quad=\prod_{\delta=+,-} \prod_{j=1}^{\left|k_{\delta}\right|}\left(\left[2 \sinh \frac{\pi}{a_{-\delta}}\left(x+i a_{\delta}\left(j_{\delta}-\theta\left(k_{\delta}\right)\right)\right)\right][i \rightarrow-i]\right)^{k_{\delta} /\left|k_{\delta}\right|}, \quad k_{\delta} \in \mathbb{Z} .
\end{aligned}
$$

[Here, $\theta(k)=1$ for $k>0$ and $\theta(k)=0$ for $k<0$.] Using (3.14) and (3.41)-(3.46), this yields the explicit formulas

$$
\begin{gathered}
M\left(a_{+}, a_{-}, b_{\alpha,-\alpha} ; x, p\right)=(-i)^{2 N_{+} N_{-}+N_{+}+N_{-}+1}\left[P_{N_{\alpha}}\left(a_{\alpha}, a_{-\alpha} ; x\right) P_{N_{\alpha}}\left(a_{\alpha}, a_{-\alpha} ; p\right)\right]^{-1} \\
\cdot K_{N_{+}, N_{-}}\left(a_{+}, a_{-} ; x, p\right), \quad \alpha=+,- \\
M\left(a_{+}, a_{-}, b_{--} ; x, p\right)=i^{2 N_{+} N_{-}+N_{+}+N_{-}} K_{N_{+}, N_{-}}\left(a_{+}, a_{-} ; x, p\right) \\
M\left(a_{+}, a_{-}, b_{++} ; x, p\right)=i^{2 N_{+} N_{-}+N_{+}+N_{-}}\left[\prod_{\alpha=+,-} P_{N_{\alpha}}\left(a_{\alpha}, a_{-\alpha} ; x\right) P_{N_{\alpha}}\left(a_{\alpha}, a_{-\alpha} ; p\right)\right]^{-1} \\
\cdot K_{N_{+}, N_{-}}\left(a_{+}, a_{-} ; x, p\right),
\end{gathered}
$$

where

$$
P_{N}\left(a_{+}, a_{-} ; x\right) \equiv \prod_{j=-N}^{N}\left[2 \sinh \frac{\pi}{a_{-}}\left(x+i j a_{+}\right)\right] .
$$

Theorem III.3: The meromorphic function $M(\Xi ; x, p), \Xi \in \mathcal{D}_{\text {hyp }}(1.7)$, satisfies 


$$
A_{\delta} M(\Xi ; x, p)=2 c_{\delta}(p) M(\Xi ; x, p), \quad \delta=+,-,
$$

and has parameter and variable symmetries

$$
\begin{gathered}
M\left(a_{+}, a_{-}, b ; x, p\right)=M\left(a_{-}, a_{+}, b ; x, p\right), \\
M(\Xi ; x, p)=M(\Xi ; p, x), \\
M(\Xi ; x, p)=M(\Xi ;-x,-p), \\
M(\Xi ; x, p)=\bar{M}(\Xi ;-x, p), \quad x, p \in \mathbb{R} .
\end{gathered}
$$

Proof: The asserted properties are clear from the definition of $M$ and from (3.58)-(3.60). [Recall that $w\left(a_{+}, a_{-}, b ; x\right)$ is symmetric under $a_{+} \leftrightarrow a_{-}$. ]

We do not know whether the function $M(\Xi ; x, p)$ admits an interpolation to all of the hyperbolic parameter domain $\mathcal{H}(1.4)$. But for the even joint $A_{\delta}$-eigenfunction,

$$
R(\Xi ; x, p) \equiv M(\Xi ; x, p)+M(\Xi ;-x, p), \quad \Xi \in \mathcal{D}_{\text {hyp }},
$$

this is the case (see Ref. 4 and papers to appear). Observe that the latter function already appeared in the integer $g$ case: One has

$$
R\left(a_{+}, a_{-},(N+1) a_{+} ; x, p\right)=R_{N}(x, p),
$$

where $R_{N}(x, p)$ is given by (2.71). [To check this, use (3.65) with $\alpha=+, N_{+}=N$ and $N_{-}=0$.]

To conclude this section, let us add one more observation on the auxiliary A $\Delta$ Os $B_{\delta}(1.21)$. Since they are only defined for $b$ of the form (1.20), we may specify their $b$-dependence by writing $B_{\delta}\left(N_{+}, N_{-}\right)$. Comparing (1.21) and (1.1), we now deduce

$$
B_{\delta}\left(N_{+}, N_{-}\right)=A_{\delta}\left(-N_{+} a_{+}-N_{-} a_{-}\right), \quad \delta=+,-
$$

This coincidence agrees with (3.66). Indeed, the latter formula says that the joint $A_{\delta}(b)$ eigenfunction $M$ for $b=-N_{+} a_{+}-N_{-} a_{-}$is proportional to the joint $B_{\delta}\left(N_{+}, N_{-}\right)$-eigenfunction $K_{N_{+}, N_{-}}$. (See also the remarks at the end of Sec. IV in I, specialized to the hyperbolic case.)

\section{THE TRIGONOMETRIC SPECIALIZATION}

At the end of the Introduction we have already delineated how various objects from the elliptic regime studied in I give rise to trigonometric counterparts. We will use the corresponding formulas (1.23)-(1.28) without further comment.

Until further notice, we restrict attention to the special choice $k=N+1 \in \mathbb{N}^{*}$ in (1.27). Then the results in Sec. II of I can be readily specialized, giving rise to functions $\Psi( \pm x, y)$ that are joint eigenfunctions of

$$
A=\frac{\sin r(x-i(N+1) \beta)}{\sin r x} T_{i \beta}+(i \rightarrow-i)
$$

[the $\mathrm{A} \Delta \mathrm{O}(1.24)$ for $b=(N+1) \beta)$ ] and $Q(1.28)$. [Indeed, the relevant trigonometric function $E(x) \mathrm{I}(2.8)$ has finite and equal limits for $\operatorname{Im} x \rightarrow \pm \infty$, entailing constancy.]

Comparing the trigonometric $\mathrm{A} \Delta \mathrm{O} A(4.1)$ to its hyperbolic counterpart $A_{-}\left((N+1) a_{+}\right)(1.1)$ one sees they are related via the substitutions

$$
a_{+} \rightarrow \beta, \quad a_{-} \rightarrow \pi / i r .
$$


Moreover, these substitutions turn the second hyperbolic $\mathrm{A} \Delta \mathrm{O} A_{+}\left((N+1) a_{+}\right)$(1.1) into $(-)^{N+1} Q$. Therefore, the joint $A_{\delta}\left((N+1) a_{+}\right)$-eigenfunctions from Sec. II can be exploited to obtain $(A, Q)$-eigenfunctions. (The latter will be shown to be essentially equal to those arising from the trigonometric specialization of Sec. II in I.)

Once more, we find it expedient to study first the pertinent eigenfunctions of the similarity transformed $\mathrm{A} \Delta \mathrm{O}$

$$
B \equiv \prod_{j=-N}^{N} \sin r(x+i j \beta) \cdot A \cdot \prod_{j=-N}^{N} \sin r(x+i j \beta)^{-1}=\frac{\sin r(x+i N \beta)}{\sin r x} T_{i \beta}+(i \rightarrow-i) .
$$

While translating our results from Sec. II to trigonometric analogs, we retain the spectral variable $y$ from Sec. II in I. As will soon become clear, this can be achieved by combining the substitutions (4.2) with

$$
\pi p / a_{-} \rightarrow \beta(y+(N+1) r)
$$

Equivalently, we can anticipate the relation to loc. cit. by taking

$$
p \rightarrow \beta y_{N} / i r
$$

where we have set

$$
y_{N} \equiv y+(N+1) r .
$$

With the above substitutions in the hyperbolic $\left(B_{+}, B_{-}\right)$-eigenfunctions $K_{N}\left(a_{+}, a_{-} ; x, p\right)$ (1.15), we obtain the trigonometric counterparts

$$
L_{N}(r, \beta ; x, y) \equiv K_{N}\left(\beta, \pi / i r ; x, \beta y_{N} / \text { ir }\right) .
$$

More specifically, this yields

$$
L_{N}(x, y)=\exp \left(i x y_{N}\right) \sum_{k, l=0}^{N} c_{k l}^{(N)}\left(q_{t}\right) \exp \left[i(N-2 k) r x+(N-2 l) \beta y_{N}\right] .
$$

Here, we are using

$$
q_{t} \equiv \exp (-\beta r)
$$

to avoid confusion with the phase factor $q$ (1.16), and the coefficients are defined by (2.2)-(2.5). Notice that in the present case all of the coefficients are real numbers, so that (4.8) entails

$$
\overline{L_{N}}(x, y)=L_{N}(-x, y), \quad x, y \in \mathbb{R} .
$$

In view of our hyperbolic result (2.1) (proved in Theorem II.1), we have

$$
B L_{N}(x, y)=2 \cosh (\beta[y+(N+1) r]) L_{N}(x, y) .
$$

Also, (1.19) translates into

$$
Q L_{N}(x, y)=-2 \cos (\pi y / r) L_{N}(x, y) .
$$

[Just as (1.19), this is immediate from (4.8), of course.] Likewise, the dual eigenfunction properties (2.48)-(2.51) become

$$
\widetilde{B} L_{N}(x, y)=2 \cos (r x) L_{N}(x, y)
$$




$$
\widetilde{Q} L_{N}(x, y)=(-)^{N} 2 \cosh (\pi x / \beta) L_{N}(x, y),
$$

where

$$
\begin{gathered}
\widetilde{B} \equiv \frac{\sinh (\beta[y+(2 N+1) r])}{\sinh (\beta[y+(N+1) r])} \widetilde{T}_{r}+\frac{\sinh (\beta[y+r])}{\sinh (\beta[y+(N+1) r])} \widetilde{T}_{-r}, \\
\widetilde{Q} \equiv \widetilde{T}_{i \pi / \beta}+\widetilde{T}_{-i \pi / \beta},
\end{gathered}
$$

with

$$
\left(\widetilde{T}_{\alpha} G\right)(y) \equiv G(y-\alpha), \quad \alpha \in \mathbb{C} .
$$

[Again, (4.14) is plain from (4.8).]

We can deduce a few more salient features from Theorem II.1. First, combining (4.7) with (2.14) and (4.10), we obtain

$$
L_{N}(x,-y-2(N+1) r)=\bar{L}_{N}(x, y), \quad x, y \in \mathbb{R} .
$$

Second, from (4.7) and (2.16) we infer

$$
L_{N}(x,-r)=L_{N}(x,-(2 N+1) r)=(-2)^{N} \prod_{k=N+1}^{2 N} \sinh (k \beta r) .
$$

Finally, (4.7) and (2.18) entail

$$
L_{N}(x,-(l+1) r)=L_{N}(x,-(2 N+1-l) r)=C_{l}^{(N)}(\cos (r x)), \quad l=0, \ldots, N .
$$

Here, $C_{l}^{(N)}(u)$ is a polynomial of degree $l$ and parity $(-)^{l}$. Moreover, this polynomial has real coefficients in view of (4.18), and one has

$$
B C_{l}^{(N)}(\cos (r x))=2 \cosh ((N-l) \beta r) C_{l}^{(N)}(\cos (r x)), \quad l=0, \ldots, N
$$

due to (4.11).

We proceed by obtaining the relation between $L_{N}(x, y)$ and the function $\mathcal{H}_{N}(x, y) \mathrm{I}(2.34)$, specialized to the trigonometric context. To this end we exploit the arguments leading from (2.52) to (2.60). Specifically, (2.52) and (2.53) remain true when $q$ is replaced by $q_{t}$. Assuming $t$ $\neq q_{t}^{-2 j}, j=1, \ldots, N$, one obtains (2.54)-(2.56). Hence the roots $\rho_{j}\left(q_{t}, t\right)$ are nonzero for $t$ $\neq q_{t}^{2 j}, j=1, \ldots, N$, and $\rho_{j}$ can be chosen equal to $q_{t}^{2 j}$ for $t=0$.

In the case at hand, we need $t=\exp \left(-2 \beta y_{N}\right)$, which entails

$$
\prod_{j=1}^{N} \rho_{j}=\prod_{j=1}^{N} \frac{\sinh (\beta[y+j r])}{\sinh (\beta[y+(N+1+j) r])}
$$

Therefore, restricting attention to $\{\operatorname{Re} y>-r\}$, we may set

$$
\rho_{j}=\exp \left(2 i r z_{j}\right), \quad z_{j}(y) \rightarrow i j \beta, \quad y \rightarrow \infty, \quad j=1, \ldots, N .
$$

Now I(2.25) yields no restriction on $\beta=-i v$, since $a=\infty$ in the trigonometric regime. Hence it follows that the functions $z_{j}(y)$ thus defined may be identified with the zero functions $z_{j}(y)$ from Sec. II of I and that the desired relation reads 


$$
L_{N}(x, y)=(4 i r)^{N} \prod_{j=1}^{N}[\sinh (\beta[y+j r]) \sinh (\beta[y+(N+1+j) r])]^{1 / 2} \cdot \mathcal{H}_{N}(x, y), \quad y \in(K, \infty) .
$$

From the trigonometric specialization of $\mathrm{I}(2.45)-\mathrm{I}(2.46)$ we also deduce that all of the zeros $z_{j}(y)$ are nonconstant.

It follows from Sec. II of I that all of the zeros $z_{1}(y), \ldots, z_{N}(y)$ belong to $i(0, \infty)$ for $y$ $\in(R, \infty)$ and $R$ large. For $N=1$ this is easily seen to be true for all $y \in(-r, \infty)$; cf. (4.22). But just as in the hyperbolic case, already for $N=2$ and a suitable choice of $\beta r$, the zeros move off the imaginary axis as $y$ decreases from $\infty$ to 0 , showing once more that the parameter $K$ is necessary. $N=2)$

To see this phenomenon happen, we use (2.9) to calculate (note $y=0$ corresponds to $t=q_{t}^{6}$ for

$$
\begin{gathered}
S_{2}\left(q ; s, q^{6}\right)=\left(q^{9}-q^{7}-q^{5}+q^{3}\right) P_{2}(q ; s) \\
P_{2}(q ; s) \equiv 1-\left(q^{4}+q^{2}+1+q^{-2}+q^{-4}\right) s+\left(q^{6}+q^{4}+2 q^{2}+2+2 q^{-2}+q^{-4}+q^{-6}\right) s^{2} .
\end{gathered}
$$

Taking $q \rightarrow q_{t}$ and letting $q_{t} \uparrow 1$, one gets $P_{2} \rightarrow 1-5 s+10 s^{2}$. Since the limit polynomial has nonreal roots, it follows that the numbers $z_{1}(0), z_{2}(0)$ are not purely imaginary for $\beta r$ small enough. [Recall that we need $s=\exp (-2 i r x)$ in the present case.]

Next, we calculate the Casorati determinant

$$
D_{N}(x) \equiv L_{N}(x+i \beta / 2, y) L_{N}(-x+i \beta / 2, y)-(\beta \rightarrow-\beta) .
$$

The argument in Sec. II of I leading to I(2.43) is easily adapted, yielding

$$
D_{N}(x)=\gamma_{N}(y) \prod_{n=-N+1 / 2}^{N-1 / 2} \sin r(x+i n \beta) .
$$

Using (4.8) with $\operatorname{Im} x \rightarrow \infty$, we now obtain

$$
\begin{aligned}
\gamma_{N}(y)= & (-2 i)^{2 N}\left[\exp \left(-\beta y_{N}\right)-\exp \left(\beta y_{N}\right)\right] \\
& \cdot\left(\sum_{l=0}^{N} c_{N l}^{(N)}\left(q_{t}\right) \exp \left[(N-2 l) \beta y_{N}\right]\right)\left(\sum_{l=0}^{N} c_{0 l}^{(N)}\left(q_{t}\right) \exp \left[(N-2 l) \beta y_{N}\right]\right) .
\end{aligned}
$$

From symmetry of the coefficients and (2.4)-(2.6) we then infer

$$
\begin{aligned}
\gamma_{N}(y) & =(-)^{N+1} 2^{2 N+1} \sinh \left(\beta y_{N}\right) \exp \left(2 N \beta y_{N}\right)(-)^{N} Q_{N}\left(e^{-2 \beta y_{N}}\right) Q_{0}\left(e^{-2 \beta y_{N}}\right) \\
& =-2^{4 N+1} \prod_{j=1}^{2 N+1} \sinh (\beta y+j \beta r) .
\end{aligned}
$$

In view of the relation (4.24), it follows that in the trigonometric case the quantity $\alpha_{N}$ in $\mathrm{I}(2.43)$ becomes

$$
\alpha_{N}=(-)^{N+1} 2 \sinh (\beta y+(N+1) \beta r) .
$$

From (4.30) we read off that $L_{N}(x, y) / L_{N}(-x, y)$ is not $i \beta$-periodic in $x$, unless $y$ equals $y_{j k}$ $\equiv-j r+i \pi k / \beta$ with $j=1, \ldots, 2 N+1$ and $k \in \mathbb{Z}$; in the latter case we readily obtain

$$
L_{N}\left(x, y_{j k}\right) / L_{N}\left(-x, y_{j k}\right)=\exp (-2 \pi k x / \beta), \quad j=1, \ldots, 2 N+1, \quad k \in \mathbb{Z} .
$$

[The formulas (4.27)-(4.32) should be compared to their hyperbolic counterparts (2.63)-(2.68).] 
Restricting attention to meromorphic $B$-eigenfunctions, it follows that the eigenspace corresponding to the eigenvalue $2 \cosh (\beta[y+(N+1) r])$ is two-dimensional over the field of $i \beta$-periodic meromorphic functions, provided $y \neq y_{j k}$; cf. Appendix B in I. (It is not hard to see that for $y$ $=y_{j k}$ this is still true; note in this connection that one need only handle the case $k=0$.) When we insist on joint $(B, Q)$-eigenfunctions with eigenvalues $(2 \cosh (\beta[y+(N+1) r]),-2 \cos (\pi y / r))$, we still obtain an infinite-dimensional eigenspace, since we can allow multipliers from the field of elliptic functions with periods $(\pi / r, i \beta)$

Next, we turn to quantum-mechanical/functional-analytic properties of the operator $B$ and its eigenfunctions. We begin by observing that the relation (4.24) can be used to define $\mathcal{H}_{N}(x, y)$ for complex $y$ with $\operatorname{Re} y>-r$ (say), and, in particular, for $y=n r, n \in \mathbb{N}$. (We have already seen that $K>0$, in general, so this is a genuine extension.) We now study the functions

$$
\psi_{n}(x) \equiv \mathcal{H}(x, n r)-\mathcal{H}(-x, n r), \quad n \in \mathbb{N},
$$

in relation to the Hilbert space

$$
\mathcal{H}_{\hat{w}} \equiv L^{2}((0, \pi / r), \hat{w}(x) d x)
$$

where

$$
\hat{w}(x) \equiv \prod_{ \pm n=1}^{N} \frac{r}{\sin r(x+i n \beta)} .
$$

First, let us note that all of the functions $\psi_{n}(x)$ belong to the dense subspace $\mathcal{O}_{1} \mathrm{I}(4.11)$ (with $N_{+}=N, N_{-}=0$, of course). Indeed, from Sec. II of I we have

$$
\mathcal{H}(i k \beta, y)=\mathcal{H}(-i k \beta, y), \quad|k| \leqslant N .
$$

[See the paragraph containing $\mathrm{I}(2.39)$.] Moreover, $\psi_{n}(x)$ is $\pi / r$-periodic ( $\pi / r$-antiperiodic) for $n$ odd (even). Hence $\psi_{n} \in \mathcal{O}_{1}$, as asserted.

Second, it is easily checked that the operator $B(4.3)$ is symmetric on $\mathcal{O}_{1}$. (One need only adapt the proof of Theorem IV.1 in I, which simplifies considerably in this case.) Now from (4.11) and (4.24) one gets

$$
B \psi_{n}=2 \cosh ([n+N+1] \beta r) \psi_{n}, \quad n \in \mathbb{N},
$$

so the functions $\psi_{n}$ are pairwise orthogonal.

Third, we combine (4.13), (4.15), and (4.24) to deduce that $\psi_{n}(x)$ satisfies the recurrence relation

$$
C_{n} \psi_{n-1}(x)+C_{n+1} \psi_{n+1}(x)=2 \cos (r x) \psi_{n}(x), \quad n \in \mathbb{N}
$$

where

$$
C_{n} \equiv\left(\frac{\sinh ([n+2 N+1] \beta r)}{\sinh ([n+N+1] \beta r)} \cdot \frac{\sinh (n \beta r)}{\sinh ([n+N] \beta r)}\right)^{1 / 2}, \quad n \in \mathbb{N} .
$$

Now for $N=0$ we have

$$
\mathcal{H}_{0}(x, y)=\exp i x(y+r), \quad \psi_{n}(x)=2 i \sin (n+1) r x, \quad C_{n}=1, \quad n \in \mathbb{N} .
$$

For $N>0$ we have $C_{0}=0$, so we deduce from (4.38) that $\psi_{0}(x)$ cannot.vanish identically. [Indeed, $\psi_{0}=0$ would entail successively $\psi_{1}=0, \psi_{2}=0, \ldots$, contradicting the $y \rightarrow \infty$ asymptotics of $\mathcal{H}_{N}(x, y)$; cf. the specialization (4.46) of $\mathrm{I}(3.41)$.] In fact, using the hyperbolic result (2.78), the function $\psi_{0}(x)$ will be explicitly determined below.

Fourth, we use (4.38) with $N>0$ to infer 


$$
\psi_{n}(x) / \psi_{0}(x)=G_{n}(\cos r x), \quad n \in \mathbb{N},
$$

where the functions $G_{n}(u)$ are polynomials of degree $n$ and parity $(-)^{n}$ with real coefficients. As a consequence, the functions $\psi_{n}, n \in \mathbb{N}$, are an orthogonal base for the Hilbert space $\mathcal{H}_{\hat{w}}$ (4.34). Of course, this entails that the operator $B$ is essentially self-adjoint on the linear span of $\psi_{0}, \psi_{1}, \ldots$, and hence on $\mathcal{O}_{1}$, too. [For $N=0$ the analogous conclusions are immediate from (4.40).]

In the following theorem we summarize some of the above findings and add some new ones. In particular, we reinterpret the three-term recurrence (4.38) in terms of the discrete difference operator

$$
D=\left(\frac{\sinh ([n+2 N+1] \beta r)}{\sinh ([n+N+1] \beta r)}\right)^{1 / 2} S\left(\frac{\sinh ([n+1] \beta r)}{\sinh ([n+N+1] \beta r)}\right)^{1 / 2}+\text { h.c. }
$$

on the Hilbert space $l^{2}(\mathbb{N})$. Here, $S$ is the right shift,

$$
(S f)_{n} \equiv\left\{\begin{array}{l}
0, \quad n=0, \\
f_{n-1}, \quad n>0,
\end{array}\right.
$$

with $f=\left(f_{0}, f_{1}, \ldots\right) \in l^{2}(\mathbb{N})$, and h.c. stands for hermitean conjugate. Clearly, $D$ is a bounded self-adjoint operator on $l^{2}(\mathbb{N})$.

Theorem IV.1: The B-eigenfunctions $\left\{(r / 2 \pi)^{1 / 2} \psi_{n}(x)\right\}_{n=0}^{\infty}$ are an orthonormal base for $\mathcal{H}_{\hat{w}}$ (4.34). The self-adjoint operator $D$ on $l^{2}(\mathbb{N})$ has a purely absolutely continuous spectrum [-2.2] with multiplicity one.

Proof: Setting

$$
N_{n} \equiv\left(\psi_{n}, \psi_{n}\right)^{1 / 2}, \quad n \in \mathbb{N}
$$

it follows from the above that the functions $\psi_{0} / N_{0}, \psi_{1} / N_{1}, \ldots$, give rise to an isometric linear map $U$ from $\mathcal{H}_{\hat{w}}$ onto $l^{2}(\mathbb{N})$. To prove that the normalization constants equal $(2 \pi / r)^{1 / 2}$, we first show that they do not depend on $n$. Indeed, consider the inner product of the recurrence relation (4.38) with $\psi_{n+1}$. By virtue of orthogonality, this yields

$$
C_{n+1} N_{n+1}^{2}=\left(\psi_{n+1}(x), \quad 2 \cos (r x) \psi_{n}(x)\right) \text {. }
$$

Now when we rewrite $2 \cos (r x) \psi_{n+1}(x)$ by using (4.38) with $n \rightarrow n+1$, then we deduce that the rhs of (4.45) equals $C_{n+1} N_{n}^{2}$. Hence we get $N_{n+1}=N_{n}$, and so our assertion follows. (This argument is probably not new, but we do not know a reference.)

Next, specializing I(3.41) to the trigonometric case, we obtain

$$
\mathcal{H}(x, y)=\mathcal{H}^{(\infty)}(x, y)+O\left(e^{-2 \beta y}\right), \quad y \rightarrow \infty,
$$

uniformly on $x$-compacts, with

$$
\mathcal{H}^{(\infty)}(x, y)=\prod_{j=1}^{N} \frac{\sin r(x+i j \beta)}{r} \cdot e^{i r x(N+1)} e^{i x y} .
$$

[This asymptotics can also be derived directly from (4.24) and (4.8).] In view of (4.33), this entails

$$
\psi_{n}(x)=\prod_{j=1}^{N} \frac{\sin r(x+i j \beta)}{r} \cdot e^{i r x(N+1+n)}-(x \rightarrow-x)+O\left(e^{-2 n \beta r}\right), \quad n \rightarrow \infty,
$$


the bound being uniform for $x \in[0, \pi / r]$. Since the inner product $\left(\psi_{n}, \psi_{n}\right)$ does not depend on $n$, we can now calculate it by using (4.48) and taking $n \rightarrow \infty$. Thus we obtain the norm formula [recall (4.35)]

$$
\left(\psi_{n}, \psi_{n}\right)=2 \pi / r, \quad n \in \mathbb{N},
$$

and so the first assertion of the theorem follows.

Now (4.38) says that $\left(\psi_{0}(x), \psi_{1}(x), \ldots\right)$ is an improper $D$-eigenfunction with eigenvalue $2 \cos r x$. More precisely, the unitary operator $U^{-1}: l^{2}(\mathbb{N}) \rightarrow \mathcal{H}_{\hat{w}}$. sets up a spectral representation for $D$ as multiplication by $2 \cos r x$ on $\mathcal{H}_{\hat{w}}$ :

$$
\left(U^{-1} D U f\right)(x)=2 \cos (r x) f(x), \quad f \in \mathcal{H}_{\hat{w}} .
$$

Thus the second assertion is plain.

We continue by determining $\psi_{0}(x)$ explicitly. To this end we note that (4.24) and (4.7) entail

$$
\psi_{0}(x)=(4 i r)^{-N} \Delta_{N}(x) \prod_{j=1}^{N}[\sinh (j \beta r) \sinh ([N+1+j] \beta r)]^{-1 / 2},
$$

where

$$
\Delta_{N}(x) \equiv K_{N}(\beta, \pi / i r ; x,-i(N+1) \beta)-K_{N}(\beta, \pi / i r ;-x,-i(N+1) \beta) .
$$

Now we use (2.14), (2.71), and (2.78) to calculate $\Delta_{N}(x)$. This yields

$$
\Delta_{N}(x)=(-)^{N} 2^{3 N+1} i \prod_{n=-N}^{N} \sin r(x+i n \beta) \cdot \prod_{j=1}^{N} \sinh (j \beta r),
$$

so that

$$
\psi_{0}(x)=r(2 i / r)^{N+1} \prod_{j=1}^{N}\left(\frac{\sinh (j \beta r)}{\sinh ([N+1+j] \beta r)}\right)^{1 / 2} \cdot \prod_{n=-N}^{N} \sin r(x+i n \beta)
$$

Comparing this explicit formula to (4.3), we deduce

$$
B=\psi_{0}(x)^{-1} A \psi_{0}(x)
$$

In view of (4.41), this entails that $A(4.1)$ can be viewed as a self-adjoint operator on the Hilbert space

$$
\mathcal{H}_{A} \equiv L^{2}\left((0, \pi / r),\left|\psi_{0}(x)\right|^{2} \hat{w}(x) d x\right),
$$

yielding an orthonorrnal base of polynomials $(r / 2 \pi)^{1 / 2} G_{n}(\cos r x)$; the $A$-eigenvalues read $2 \cosh ([n+N+1) \beta r)$ [cf. (4.37)], and the polynomials are uniquely determined by the recurrence (4.38) and $G_{0}(u)=1$ (save for $N=0$; cf. below).

As already pointed out in Ref. 5 [cf. the paragraph in Ref. 5 containing Eq. (3.84)], the orthogonal polynomials thus obtained are not new: They are $q$-Gegenbauer polynomials generalizing the integer $g$ Gegenbauer polynomials arising from the trigonometric specialization of the Lamé operator I(1.1). These $q$-Gegenbauer polynomials were studied in considerable detail by Askey and Ismail, ${ }^{6}$ their parameters are related to ours via

$$
q_{\mathrm{AI}}=q_{t}^{2}=\exp (-2 \beta r), \quad \beta_{\mathrm{AI}}=\exp (-2 g \beta r), \quad \lambda_{\mathrm{AI}}=g .
$$


As far as we know, the two representations we have exploited to derive some important features of the integer $g$ polynomials are new. In this connection we should also point out that the pertinent weight function integral is immediate from the above. Indeed, from (4.49) we have, in particular, $\left(\psi_{0}, \psi_{0}\right)=2 \pi / r$. Hence (4.54) and (4.35) yield the integral

$$
\frac{1}{2 \pi} \int_{0}^{\pi} d y \sin y \prod_{n=-N}^{N} \sin (y+i n \alpha)=\frac{1}{4^{N+1}} \prod_{j=1}^{N} \frac{\sinh ([N+1+j] \alpha)}{\sinh (j \alpha)}, \quad N \in \mathbb{N}, \quad \alpha>0,
$$

as a corollary.

In the remainder of this section we study the case $b=k \beta$ with $-k \in \mathbb{N}$. Since we intend to compare the insights obtained for these parameters to the state of affairs at the elliptic level, we follow the relevant part of Sec. IV in I [starting with the paragraph containing I(4.42)] to a large extent. We first need some preparations, however.

First, in keeping with the notation adopted for the elliptic case, we denote the $A((N+1) \beta)$-eigenfunctions corresponding to $\mathcal{H}_{N}(x, y)$ by $\Psi(x, y)$. Thus, we have

$$
\Psi(x, y)=\mathcal{N} \prod_{j=-N}^{N} \sin r(x+i j \beta)^{-1} \cdot \mathcal{H}_{N}(x, y), \quad b=(N+1) \beta,
$$

so that we get the asymptotics

$$
\Psi(x, y) \sim c(x) \exp (i x y), \quad y \rightarrow \infty,
$$

with

$$
c(r, \beta,(N+1) \beta ; x)=\mathcal{N} \prod_{j=0}^{N} \sin r(x-i j \beta)^{-1} \cdot \exp i(N+1) r x .
$$

(As before, the symbol $\mathcal{N}$ is used to denote normalization constants.)

Second, the trigonometric $c$-function occurring here equals, more generally,

$$
c(r, \beta, b ; x)=\frac{G(r, \beta ;-x-i \beta / 2)}{G(r, \beta ;-x+i b-i \beta / 2)},
$$

where $G(r, \beta ; z)$ is the generalized trigonometric gamma function from Ref. 2; just as in the elliptic case, the weight and scattering functions are then given by $\mathrm{I}(1.27)$ and $\mathrm{I}(1.28)$. [Compare also I(1.24)-I(1.26) with (4.60)-(4.62).]

We are now prepared to follow our elliptic reasoning. Denoting $B(4.3)$ by $B(N)$, and comparing this $\mathrm{A} \Delta \mathrm{O}$ to $A(b)(1.24)$, we obtain

$$
A(-N \beta)=B(N), \quad N \in \mathbb{N} .
$$

This equality is the trigonometric counterpart of the elliptic relation $\mathrm{I}(4.42)$ with $N_{-}=0$ and $\delta$ $=-$; the prefactor $r_{-}$is absent here, since we omitted the prefactor $\exp (-b r)$ occurring in $\mathrm{I}(1.15)$; cf. (1.24). (As will be explained at the end of this section, in the trigonometric case this omission is not motivated solely by a desire to avoid clutter from constants.)

Due to (4.63), (4.11), and (4.24) we now have

$$
A(-N \beta) \mathcal{H}_{N}( \pm x, y)=2 \cosh (\beta[y+(N+1) r]) \mathcal{H}_{N}( \pm x, y)
$$

which is the analog of I(4.46) with $N_{-}=0, \delta=-$. Similarly, I(4.48) and I(4.50) specialize to

$$
\Psi(x, y)=\mathcal{N} \mathcal{H}_{N}(x, y-(2 N+1) r), \quad b=-N \beta, \quad \operatorname{Re} y>2 N r .
$$


[Note that (4.24) and (4.20) entail that $\Psi(x, y)$ becomes singular for $y=(N+j) r$ with $\pm j$ $=1, \ldots, N$.] This guarantees that $(4.60)$ holds true, with

$$
c(-N \beta ; x)=\mathcal{N} \prod_{j=1}^{N} \sin r(x+i j \beta) \cdot \exp -i N r x
$$

[This formula agrees with the pertinent specializations of $\mathrm{I}(4.52)$ and (4.62); cf. also Proposition III.14 in Ref. 2.]

The upshot is that we have now obtained eigenfunctions $\Psi(b ; \pm x, y)$ of $A(b)$ [and of $Q$ (1.28), of course] for $b=k \beta, k \in \mathbb{Z}$. Denoting the eigenvalues by $E(y)$, they have asymptotics

$$
E(y) \sim e^{b r} e^{\beta y}, \quad y \rightarrow \infty, \quad b=k \beta, \quad k \in \mathbb{Z},
$$

in accord with $\mathrm{I}(1.19)$ for $\delta=-$. [Recall that we omitted the factor $\exp (-b r)$ in $\mathrm{I}(1.15)$ for the trigonometric $\mathrm{A} \Delta \mathrm{O}(1.24)$.]

In contrast to the elliptic and hyperbolic cases, the subset $\mathcal{D}_{\text {trig }}(1.27)$ is not dense in the trigonometric parameter domain $\mathcal{T}(1.23)$. Therefore, our results have no direct implications for the existence and properties of joint $(A(b), Q)$-eigenfunctions $\Psi(x, y)$ for arbitrary $b \in \mathbb{R}$. But we can shed more light on the orthogonality question for the elliptic case by specializing to the $b$-values $-N \beta, N \in \mathbb{N}$, and studying the analogous trigonometric question. Then we are dealing with the Hilbert space of square-integrable functions on $(0, \pi / r)$ w.r.t. the measure $w(r, \beta,-N \beta ; x) d x$; due to (4.66) and the relation $w(x)=1 / c(x) c(-x)$, the weight function is proportional to $\hat{w}(x)$ (4.35), so we may as well use $\mathcal{H}_{\hat{w}}$ to study orthogonality.

First, we observe that due to (4.65) the functions

$$
\zeta_{k}(x) \equiv \Psi(x, k r)-\Psi(-x, k r), \quad k \geqslant 2 N+1, \quad b=-N \beta,
$$

are proportional to the functions $\psi_{k-2 N-1}$ given by (4.33). Thus they yield an orthogonal base of eigenvectors of $A(-N \beta)$. But in this case we can actually rule out that for $N>0$ the even combinations

$$
\chi_{k}(x) \equiv \Psi(x, k r)+\Psi(-x, k r), \quad k \geqslant 2 N+1, \quad b=-N \beta
$$

are orthogonal in $\mathcal{H}_{\hat{w}}$. This entails that the elliptic generalizations for $b=-N_{\delta} a_{\delta}, N_{\delta}>0$, are not orthogonal as well [as announced below I(4.57)]. In the following theorem we prove not only nonorthogonality, but also an unexpected completeness property.

Theorem IV.2: For all $N \in \mathbb{N}$ the functions $\chi_{k}(x)$ (4.69) satisfy

$$
A(-N \beta) \chi_{k}(x)=2 \cosh ([k-N] \beta r) \chi_{k}(x), \quad k \geqslant 2 N+1 .
$$

Now let $N \in \mathbb{N}^{*}$. Then the functions $\left\{\chi_{k}(x)\right\}_{k=2 N+1}^{\infty}$ are total, but not pairwise orthogonal in the Hilbert space $\mathcal{H}_{\hat{w}}$ (4.34).

Proof: The first statement follows from (4.64) and (4.65). In order to prove the second one, we begin by noting that the functions $\chi_{k}(x)$ satisfy the recurrence relation

$$
C_{n} \chi_{2 N+n}(x)+C_{n+1} \chi_{2 N+n+2}(x)=2 \cos (r x) \chi_{2 N+n+1}(x), \quad n \in \mathbb{N},
$$

with $C_{n}$ given by (4.39). [Indeed, this follows in the same way as (4.38).] Since $C_{0}=0$ and $C_{n}$ $>0$ for all $n \in \mathbb{N}^{*}$, this entails as before that $\chi_{2 N+1}(x)$ is not identically 0 and that

$$
\chi_{2 N+n+1}(x) / \chi_{2 N+1}(x)=G_{n}(\cos r x), \quad n \in \mathbb{N}
$$

cf. (4.41). 
Since $G_{n}(\cos r x)$ is a polynomial of degree $n$ in $\cos r x$, it now follows from (4.72) that the linear span of the functions $\chi_{2 N+1}, \chi_{2 N+2}, \ldots$, is dense in $\mathcal{H}_{\hat{w}}$. Thus, it remains to show that they are not pairwise orthogonal. We now prove this by deriving a contradiction from the assumption of pairwise orthogonality.

Indeed, this assumption entails (by virtue of the reasoning in the proof of Theorem IV.1) that the polynomials $(r / 2 \pi)^{1 / 2} G_{n}(\cos r x)$ are an orthonormal base for the Hilbert space

$$
\widetilde{\mathcal{H}}_{A} \equiv L^{2}\left((0, \pi / r),\left|\eta_{0}(x)\right|^{2} \hat{w}(x) d x\right),
$$

where

$$
\eta_{0}(x) \equiv \mathcal{H}_{N}(x, 0)+\mathcal{H}_{N}(-x, 0)
$$

Since we have already proved that these polynomials have this property w.r.t. the Hilbert space $\mathcal{H}_{A}$ (4.56), it easily follows that $\left|\psi_{0}(x)\right|^{2}$ and $\left|\eta_{0}(x)\right|^{2}$ are equal for $x \in[0, \pi / r]$. Now this amounts to the real part of $\overline{\mathcal{H}}_{N}(x, 0) \mathcal{H}_{N}(-x, 0)$ being 0 for $x \in[0, \pi / r]$, so using (4.24) we infer $\operatorname{Re} \bar{L}_{N}(x, 0) L_{N}(-x, 0)$ vanishes for $x \in[0, \pi / r]$. Recalling (4.10), this entails $\operatorname{Re}\left(L_{N}(x, 0)^{2}\right)=0$ for real $x$. But an inspection of (4.8) reveals that the function $L_{N}(x, 0)^{2}$ is of the form $\sum_{m=2}^{4 N+2} a_{m} \exp i m r x$, with $a_{m} \in \mathbb{R}$. Thus, we infer $L_{N}(x, 0)$ vanishes identically. Since this entails $\psi_{0}(x)=0$, we finally arrive at the desired contradiction.

The alert reader will have noted that we excluded the choice $N=0$ from consideration. Indeed, from (4.65) and the $N=0$ formula (4.40), we have $\Psi(x, y)=\mathcal{N} \exp i x y$. Thus, the functions $\zeta_{k}(x)$ (4.68) and $\chi_{k}(x)$ (4.69) are proportional to $\sin k r x$ and $\cos k r x$, respectively, with $k=1,2, \ldots$. Moreover, $\mathcal{H}_{\hat{w}}$ reduces to $L^{2}((0, \pi / r), d x)$. Now, as before, the functions $\zeta_{1}, \zeta_{2}, \ldots$, are an orthogonal base. But clearly the functions $\chi_{1}, \chi_{2}, \ldots$, are also pairwise orthogonal, and they are not complete in $\mathcal{H}_{\hat{w}}$, since they are all orthogonal to the constant functions!

At first sight, this seems to contradict our previous reasoning. In fact, however, there is a subtle, but decisive difference with the case $N>0$ : The pertinent recurrence coefficients $C_{n}, n$ $\in \mathbb{N}$, are equal to 1 including $C_{0}$, whereas $C_{0}$ vanishes for $N>0$ [cf. (4.39)]. Hence it does not follow that (4.72) yields polynomials, and indeed the functions $\cos (n+1) r x / \cos r x$ are not polynomials in $\cos r x$.

We also observe that the $N=0$ recurrence is obeyed both by the second-kind Tchebichev polynomials $\sin (n+1) r x / \sin r x$ and by the first-kind ones $\cos n r x, n \in \mathbb{N}$. The latter can be used to define $A(0)$ as a self-adjoint operator on $L^{2}((0, \pi / r), d x)$, whereas the former are equal to the above $q_{t}^{2}$-Gegenbauer polynomials for $b=\beta$ [and as such were used to turn $A(\beta)$ into a selfadjoint operator on $\left.L^{2}\left((0, \pi / r), \sin ^{2}(r x) d x\right)\right]$.

To conclude this section, we present some more observations on the relation between the cases $b=(N+1) \beta$ and $b=-N \beta$. When we transform the $A(b)$-eigenfunctions $\Psi(x, y)$ to functions $\mathcal{F}(x, y)$ by using $\mathrm{I}(1.29)$ [with $w(x)$ the trigonometric $w$-function $w(r, \beta, b ; x)]$, then the functions $\widetilde{\mathcal{F}}(x, y)$ for the $b$-values $-N \beta$ are related to the functions $\mathcal{F}(x, y)$ for the $b$-values $(N$ $+1) \beta$ via

$$
\tilde{\mathcal{F}}(x, y)=\chi \mathcal{F}(x, y-(2 N+1) r), \quad \operatorname{Re} y>2 N r,
$$

with $\chi$ a phase. [This follows by combining (4.59) and (4.65) with the $c$-function formulas (4.61) and (4.66).]

This relation is in agreement with the identity

$$
H(-b)=H(b+\beta),
$$

satisfied by the Hamiltonian $H(b)$ (1.25). We have omitted the factor $\exp (-b r)$ present in the elliptic counterpart $H_{-} \mathrm{I}(1.12)$, since the symmetry property $\mathrm{I}(1.13)$ does not admit a trigonometric specialization. Because we have done so, the symmetry property (4.76) appears instead. Note 
that this invariance property at the relativistic level turns into $g \rightarrow 1-g$ invariance at the nonrelativistic level; cf. I(1.1). By contrast, the invariance property $\mathrm{I}(1.13)$ has no nonrelativistic counterpart.

Let us observe finally that - again in contrast to the elliptic case-the A $\Delta \mathrm{Os} H\left(b_{1}\right)$ and $H\left(b_{2}\right)$ are proportional (in fact, equal) only when $b_{2}=b_{1}$ and $b_{2}=-b_{1}+\beta$. Of course, this easily verified assertion assumes that we restrict attention to $\mathcal{T}(1.23)$, as we have done throughout this section.

\section{ACKNOWLEDGMENT}

This work was partly supported by the Netherlands Organisation for the Advancement of Research (NWO).

${ }^{1}$ S. N. M. Ruijsenaars, "Generalized Lamé functions. I. The elliptic case,” J. Math. Phys. 40, 1595-1626 (1999).

${ }^{2}$ S. N. M. Ruijsenaars, " First order analytic difference equations and integrable quantum systems," J. Math. Phys. 38, 1069-1146 (1997).

${ }^{3}$ S. N. M. Ruijsenaars, "Action-angle maps and scattering theory for some finite-dimensional integrable systems. I. The pure soliton case," Commun. Math. Phys. 115, 127-165 (1988).

${ }^{4}$ S. N. M. Ruijsenaars, "Systems of Calogero-Moser type," to be published in Proceedings of the 1994 Banff summer school 'Particles and fields', edited by G. Semenoff and L. Vinet.

${ }^{5}$ S. N. M. Ruijsenaars, "Finite-dimensional soliton systems," in Integrable and Superintegrable Systems, edited by B. Kupershmidt (World Scientific, Singapore, 1990), pp. 165-206.

${ }^{6}$ R. Askey and M. E.-H. Ismail, "A generalization of ultraspherical polynomials," in Studies in Pure Mathematics, edited by P. Erdös (Birkhäuser, Boston, 1983), pp. 55-79. 